Review

\title{
Optical Phenomena in Mesoscale Dielectric Particles
}

\author{
Oleg V. Minin and Igor V. Minin *
}

School of Nondestructive Testing, Tomsk Polytechnic University, Lenina Ave. 30, 634050 Tomsk, Russia; ovminin@tpu.ru

* Correspondence: ivminin@tpu.ru or prof.minin@gmail.com

Citation: Minin, O.V.; Minin, I.V. Optical Phenomena in Mesoscale Dielectric Particles. Photonics 2021, 8 , 591. https://doi.org/10.3390/ photonics 8120591

Received: 24 November 2021 Accepted: 16 December 2021 Published: 19 December 2021

Publisher's Note: MDPI stays neutral with regard to jurisdictional claims in published maps and institutional affiliations.

Copyright: (c) 2021 by the authors. Licensee MDPI, Basel, Switzerland. This article is an open access article distributed under the terms and conditions of the Creative Commons Attribution (CC BY) license (https:// creativecommons.org/licenses/by/ $4.0 /)$.

\begin{abstract}
During the last decade, new unusual physical phenomena have been discovered in studying the optics of dielectric mesoscale particles of an arbitrary three-dimensional shape with the Mie size parameter near $10(q \sim 10)$. The paper provides a brief overview of these phenomena from optics to terahertz, plasmonic and acoustic ranges. The different particle configurations (isolated, regular or Janus) are discussed, and the possible applications of such mesoscale structures are briefly reviewed herein in relation to the field enhancement, nanoparticle manipulation and super-resolution imaging. The number of interesting applications indicates the appearance of a new promising scientific direction in optics, terahertz and acoustic ranges, and plasmonics. This paper presents the authors' approach to these problems.
\end{abstract}

Keywords: mesoscale particle; Mie size parameter; optical effect

\section{Introduction}

Mesoscale dielectric particles are mesostructures comprising both wavelength-scaled particles and particle chains or arrays. These particles are made of low loss dielectric materials having relatively low refractive index, namely the refractive index less than two. The exact solution of the Maxwell equation for plane wave scattering over a homogeneous dielectric sphere is described by the Mie equations [1], which include the size parameter $q=2 \pi R / \lambda$, where $R$ is the sphere radius, $\lambda$ is the emission wavelength depending on the particle refractive index $n$. The field structures with the size parameter $q \sim 10$ lying between the nano $(q \sim 1)$, wave and geometric optics $(q>100)$ have not been studied so far. However, in the last decade, new, unusual phenomena have been discovered in optics of arbitrary three-dimensional dielectric particles at the mesoscale level and gratings with $q \sim 10$ parameter. It is important to identify these phenomena. Below we present the authors' approach to these problems.

\section{Optical Optical Whirlpools, Nano-Vortices, Optical Hearts}

The anomalous light scattering possesses the number of interesting and unusual properties such as a complex structure of the near field, which may include optical vortices, optical whirlpool and other specific traits of the nanoscale level [2]. For example, the term optical whirlpool was introduced in [3] by Bashevoy et al., who show that near the plasmonic resonance of the metal particle, nanosized optical vortices are observed, which resemble an optical whirlpool. Luk'yanchuk et al [4]. describe the optical vortices with the specific heart size, significantly lower than the diffraction limit for particles with a low size parameter $q<1$. It is also found that near-field optical vortices appear at points matching the symmetric quadrupole resonance and forward and back scattering.

Another interesting effect connected with the optical vortices is a possibility of threedimensional subwavelength confinement of light outside the dielectric particle with $q \sim 10$ parameter, known as a photonic jet. This is associated with the vortex formation in the near field, namely the field squeeze due to the optical vortices occurring at the particle boundary, near the formation of the photonic jet [5]. 
Based on the Lorenz-Mie theory, it was found in [6] that spherical mesoscale particles with definite size parameters could cause substantially great field strength in singularities and form two extreme hotspots near the particle poles. Thus, the increase in the field intensity in the hotspots corresponded to 438 and 514 values for the size parameters of $q=22.24159$ and $q=28.64159$ of the Teflon sphere, respectively. Additionally, a surprisingly large circulation was discovered for the three-dimensional Poynting vector in the form of heart (see Figure 1), which was impossible to predict by a conventional two-dimensional analysis.

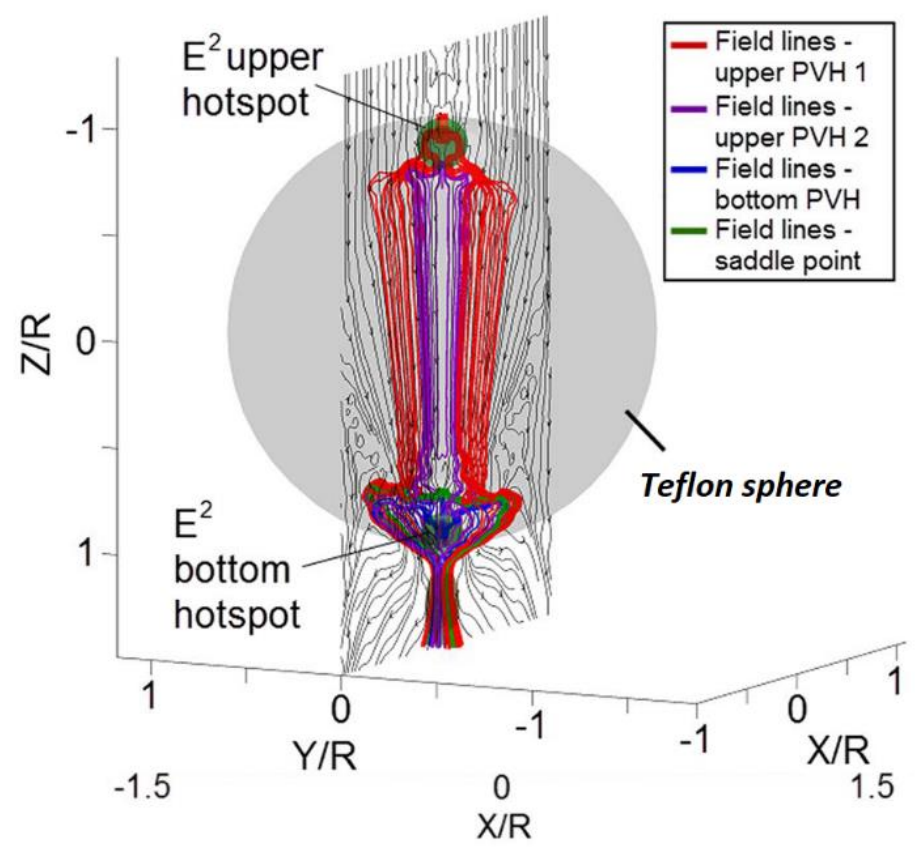

Figure 1. Three-dimensional view of Poynting vectors at critical points. Particle size parameter: $q=22.24159$. PVH - Poynting vector hotspot. Adapted from [6].

These new physical phenomena allow explaining such interesting effects as the formation of the photonic jet and hotspots.

\section{Fano Resonances in Dielectric Mie Resonance Mesophotonics}

The crucial role in the formation of Fano resonances play magnetic dipole resonances of isolated dielectric particles. The magnetic dipole mode of the dielectric particle excited at the wavelength of the magnetic resonance can be stronger than the electric dipole response, thereby contributing to the scattering effectiveness. Luk'yanchuk et al. [7] show that low-scattering mesoscale dielectric microspheres can produce high-order Fano resonances associated with the internal Mie modes. It follows from this that the regions associated with such a superresonance can be located inside a spherical particle and the field structure has no whispering gallery mode. These resonances observed at a certain size parameter yield the magnetic field enhancement of the order of magnitude of $10^{4}-10^{7}$. In this respect, super-resonances demonstrate the appearance of the magnetic photonic jets and giant magnetic fields in dielectric microspheres with high refractive index of $\sim 4$, that may be attractive to many photonic applications.

Yue et al. [8] identified the super-enhancement focusing of Teflon spheres with the certain size parameters and the refractive index $n<2$, which rendered the field enhancement about 4000 times stronger than that of the downward radiation and demonstrated the possibility of overcoming the diffraction limit despite the high sensitivity to losses in the particle material. The Teflon spheres were characterized by a unique arrangement of hotspots at the poles of the sphere and were stipulated by a specific behavior of the Mie modes [8]. The manifestation of the super resonance effect is clearly seen in Figure 2. This 
figure also shows the effect of transformation of hot spots at the poles of the sphere in the absence of the photonic jet losses when small losses are introduced into the sphere material. With increasing losses in the sphere material, the maximum intensity of the electric and magnetic fields was compared for the size parameter $q=22.24159$. At $q=28.64159$, the magnetic field intensity was approximately two times higher than that of the electric field, in the absence of the particle material losses. The size decrease in the field localization (hotspots) down to less than the diffraction limit was observed after the introduction of small losses into the sphere material, which could be even less than in the ideal case of the particle material without losses (Figure 2b).
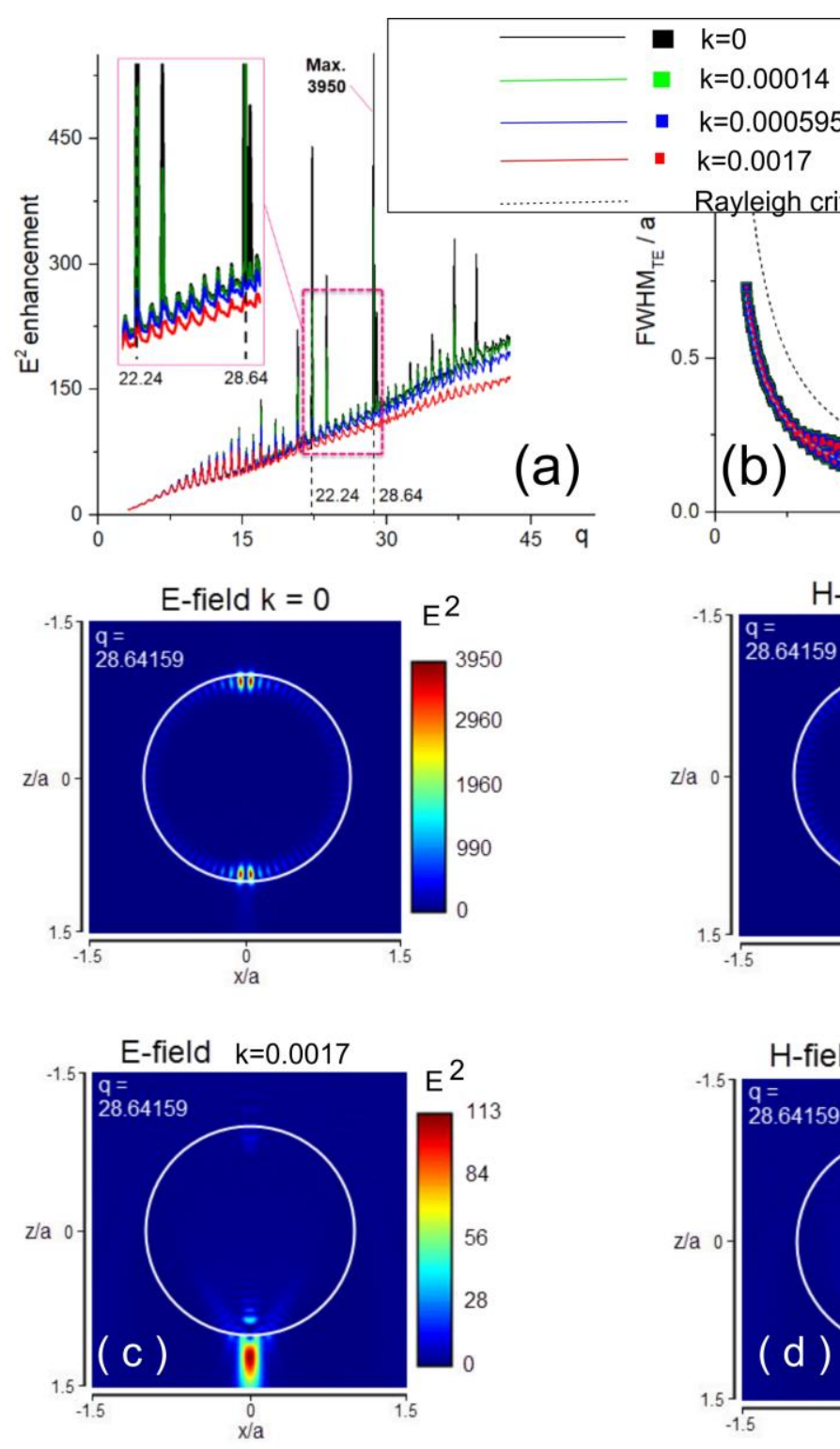

- $\mathrm{k}=0.0017$
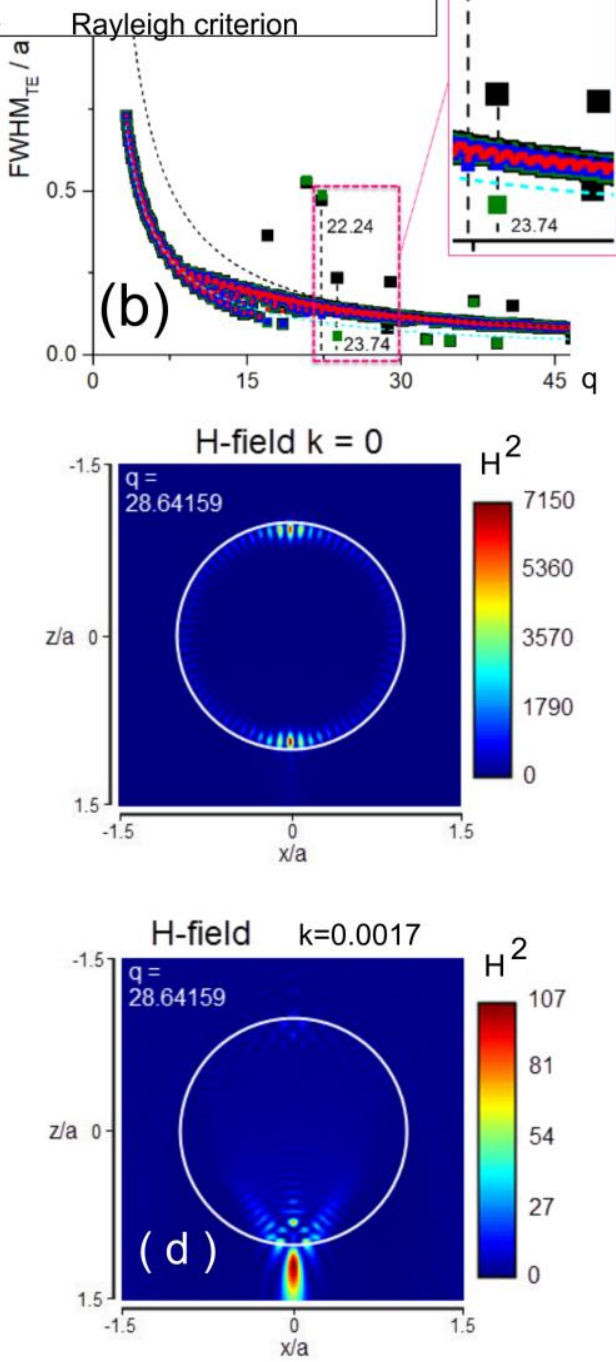

Figure 2. Distribution of the maximum field intensity for a spherical Teflon particle (refractive index $n=1.43$ ) depending on the size parameter $q$ at different absorption coefficient of the particle material $k$ (a). Dependence of the field localization width on the size parameter $q$ at different absorption coefficient in the particle material $k(\mathbf{b})$. Electric (c) and magnetic (d) field intensities at the resonance value of the size parameter $q=28.64159$ and absorption indices $k=0$ and $k=1.7 \times 10^{-3}$. Adapted from [8]. 


\section{Giant Magnetic Field Generation in Mesoscale Particles}

A new physical effect of the optical super-resonance in mesoscale dielectric spheres is conditioned by the high-order Fano resonance $[7,8]$ and can become a new method of achieving super-high magnetic fields. This is conditioned by the following factors. The high-order Fano resonances in such particles are characterized by the high degree of the field localization, that exceeds the diffraction limit both inside the particle and on its surface. The latter is associated with the formation of regions having high values of the local wavenumber vectors by analogy with the superoscillation effects $[9,10]$. In accordance with this theory, the local wavenumber vector is a local phase gradient, viz. $K_{1}=\Phi=\nabla E / E$. The high $K_{1}$ values can be created, for example, in free-space optics, including vortices and point vortices. This results from the uncertainty principle $\Delta E \Delta t \geq \hbar / 2$, which in terms of the number of photons and photon phase can be reformulated as $\Delta N \Delta \Phi \geq 1 / 2$. Differentiation of this relation results in $K_{1} \sim \nabla N / N^{2}$, i.e., the high wavenumbers can be reached in the vicinity of the optical vortex representing a singularity (zero-intensity point) with the phase of the field circulating round this point [7]. For example, for superresonance condition (Figure 2) the local ratio of wave vectors in singular points near the outer surface of a particle can reach $K_{0} / K_{1} \sim 10^{-2}$, where $K_{0}$ is a wavevector of incident wave. So the giant magnetic fields can be created inside the dielectric particle due to creating subwavelength optical vortices with large phase gradients in the vicinity of singularities.

Optical vortices occur in dielectric mesoscale particles, when the size parameter $q$ exceeds a certain value depending on its high refractive index [5]. Accordingly, the ring currents create magnetic fields in accordance with the Biot-Savart law. In accordance with this law, the coil radius must be reduced to increase the $H$ field amplitude. Thus, the minimum radius of the respective equivalent coil is limited by the size of the order of one wavelength. Inside the dielectric particle, the magnetic field can be enhanced by more than four orders, resulting in the magnetic field of about $10^{5}$ tesla, which approaches the interatomic magnetic fields [7]. The disadvantages of the high magnetic field generated by higher order resonances are highly sensitive to the mesoscale particle material losses. Note that such a magnetic field is comparable with magnetic cumulative generators [11,12]. With such magnetic fields, magnetic nonlinear optics effects can be expected when the refractive index is changed by the magnetic effects only. This magnetic nonlinear optics can however be implemented under two conditions: (1) rather low dissipation, (2) magnetic nonlinear response significantly exceeds the electric nonlinear response due to nonlinearity, viz. $\varepsilon=\varepsilon(E)[7]$.

An effect similar to the resonances noted above is also observed for particles of a different shape, other than a sphere or a cylinder. For example, for a cubic particle with a side of about $2 \lambda(q \sim 2 \pi)$ and $n=1.59$, for linear polarization and under conditions of the geometric resonance, the hot spot intensity $E^{2}$ near the shadow side of the cube is almost 400 higher than that of the plane wave incident on the cube (for a sphere with the size parameter $q \sim 5.38$ and the overlapping of multiple resonance modes $\left.E^{2}(\max ) \sim 40\right)$. It should be noted that in dielectric mesoscale particles, the maximum field intensity is close to the particle boundary in contrast to plasmonic particles, where the maximum field intensity is observed on its surface. However, a detailed analysis of the electric and magnetic field structures for non-spherical particles is beyond the scope of this paper and will be discussed elsewhere.

These effects of high-order Fano super-resonances are very attractive for such promising directions as, for example, the improvement of absorption effects, ablation induced by the magnetic pressure, and others.

\section{Photonic Nanojets}

The term photonic nanojet was introduced by Chen et al. [13], although this effect was known before, e.g., [14-17]. In accordance with the theory of scattering on a cylindrical or spherical particles, e.g., $[14,18]$, the optical field both inside and outside the low-absorbing particle illuminated by a light wave is characterized by the spatial focus areas, called inner 
and outer focuses. These are associated with the surface curvature of the spherical particle leading to deformations of the phase wavefront incident on the particle. In this case, the spherical or cylindrical particle plays the role of a refractive lens, which focuses the light wave within the subwavelength volume [19]. At the same time, if the particle is in the converging wave area, the focusing area shifts toward the particle center and can even be located inside it [20]. Moreover, in illuminating the particle by the structured fields, for example, a circularly polarized vortex beam, a reverse energy flow occurs in the forming photonic jet in the focal plane, that depends both on the topological charge of the optical vortex and the size parameters of spherical particles [21].

The complex interaction between the aberrated areas of the wavefield in the dielectric particle depends on the particle shape, its material properties, Mie size parameter, etc. At the determined proportion of the particle parameters (optical contrast, size parameter), one of the particle inner focuses moves to its outer boundary, thereby forming a strongly localized field, namely photonic jet, near the shadow-side surface and outside the particle.

Chen et al. [13], reported that the photonic jet could propagate to a distance exceeding the wavelength $\lambda$ after its exit from the dielectric cylinder or microsphere with the diameter over $2 \lambda$, fabricated without material loss [22]. The photonic jet characterized by the beam waist could be lower than the classical diffraction limit [19], namely about one third of the illuminated light wavelength. Note that in flat diffraction optics, such a size of the beam waist was achieved at a focal length less than the wavelength $[23,24]$. However, in general cases, the transverse size of the photonic jet could be either smaller or larger than the diffraction limit.

It should be noted that the photonic jet occurs in the space region, where near-field scattering plays a significant part (evanescent fields). Therefore, the agreement between the geometric optics approximation and the strict electromagnetic theory is less accurate for the particle size within the order of the light wavelength since the evanescent field is neglected in geometric optics [25]. The near-field region is usually located at distances not exceeding several particle diameters and is characterized by a notable contribution of the radial component of the optical field to this region. In turn, this condition restricts the size range of the dielectric particles, which is of the order of several wavelengths and even equals the light wavelength [5,26], i.e., has mesoscale sizes. Hence, the specific value of the incident wavelength is not critical until mesoscale conditions are true.

The phenomenon of the photonic jet is not specific to dielectric mesoscale axisymmetrical 2D (cylindrical) or 3D (spherical) particles. In [27,28], it was shown for the first time that the photonic jet could be formed by nonspherical and asymmetrical dielectric mesoscale particles of an arbitrary shape, both in transmission and reflection modes. That provided better understanding of the photonic jet properties and possible ways of adjustment of such structured wave beams. On the other hand, the particle axial asymmetry and nonsphericity induced new optical effects. In particular, the focusing properties of cubic particles could be explained by the edge diffraction phenomenon in contrast to the refractive phenomena for spherical particles [26].

A range of the photonic jet applications is currently rather wide. Since the early 2000s, it has been used for optical manipulations [29] and can also be applied to detect and manipulate the biological objects [30], biomedicine [31,32], micro and macro manipulations [33], optical tweezers [34,35], Raman spectroscopy [36,37], microscopy [38,39] and many others.

\section{Photonic Jet: Electric or Magnetic?}

It is interesting to note, that for dielectric particles with some size parameters, the photonic jet is magnetic rather than electric [5]. In other words, during the photonic jet formation, the magnetic field enhancement can be stronger than that which corresponds to the electric field. Figure 3a,b [5] depicts the electric-field $E^{2}$ distribution with the field enhancement $H^{2}$ for a spherical particle, the high refractive index $n \sim 1.5$ and the size parameter $q \sim 10$. One can see that in the localized radiation area, the maximum magneticfield intensity is about two times higher than the electric-field intensity. 

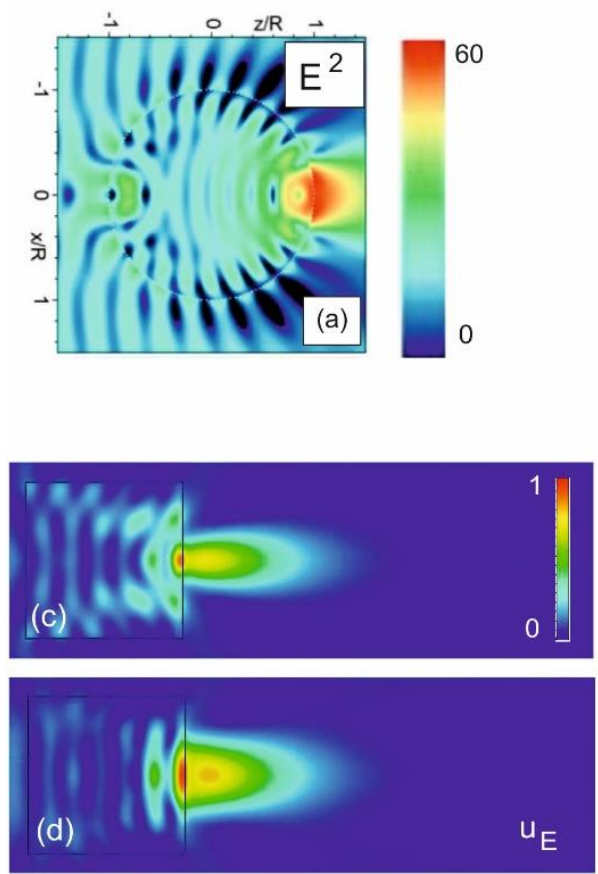
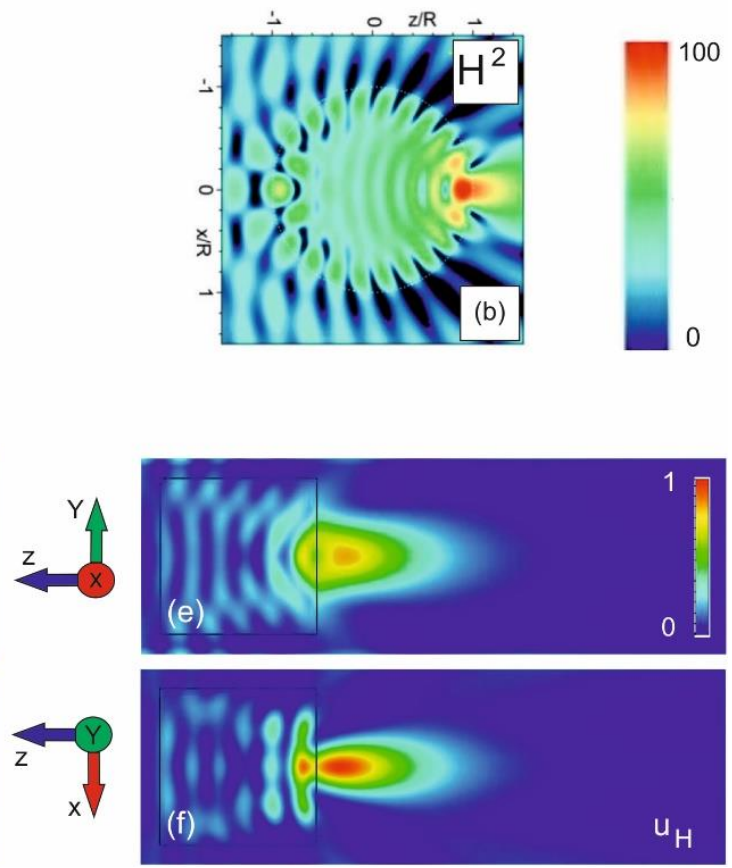

Figure 3. Intensity distribution in $\{x-z\}$ plane: electric field $E^{2}(\mathbf{a})$, magnetic field $H^{2}(\mathbf{b})$, for spherical particle $(n \sim 1.5$ and $q \sim 10)$. Adapted from [15]. The temporally averaged normalized values of the electric $\mathrm{u}_{\mathrm{E}}(\mathbf{c}, \mathbf{d})$ and magnetic $\mathrm{u}_{\mathrm{H}}(\mathbf{e}, \mathbf{f})$ energy densities for a low loss cubic Teflon particle illuminated by a plane wave with linear polarization $E_{\mathrm{x}}$.

Usually, the electric dipole transitions are $10^{4} \sim 10^{5}$ stronger than the magnetic dipole transitions. However, unlike plasmonic particles, the first resonance of the dielectric spherical particles is a magnetic dipole resonance, which occurs when the light wavelength in the particle equals the diameter, viz. $\lambda / n \sim 2 R$. In this case, the electric field polarization is antiparallel at the opposite boundaries of the sphere, that provides a strong connection with circulating displacement currents, whereas at the center, the magnetic field vibrations are directed up and down [40].

Figure $3 c-f$ depicts the temporally averaged values of the electric (Figure $3 c, d$ ) and magnetic (Figure 3e,f) energy densities for a cubic Teflon particle with the refractive index $n \sim 1.45$ and the size parameter $q \sim 2 \pi$ (cube size is $2 \lambda \times 2 \lambda \times 2 \lambda$ ). One can see that the maximum density of the electric energy is located inside the particle, near its shadow surface, while the maximum density of the magnetic energy is located in the photonic jet region. In this case, the photonic jet is magnetic rather than electric.

The magnetic field enhancement earlier identified for nanoparticles with the high refractive index [41,42] can therefore be observed for particles with the relatively low $(n<2)$ refractive index, if their size is larger than the wavelength $(R>\lambda)$, which is typical for the range of the photonic jet existence [5] for polarized [15] and unpolarized [43] illuminating light source. This property can be used, for example, for the radiation enhancement with the magnetic dipole transition [44].

\section{Anomalous Apodization Effect}

It is known that the amplitude mask apodization of the lens center reduces the transverse size of the focused beam, but the intensity in the focus lowers owing to blocking the part of the incident wave [45]. The same is also observed for spherical mesoscale particles $[46,47]$.

The anomalous apodization effect (size reduction in the photonic jet beam waist at its intensity increase) was first discovered for mesoscale particles of various nonspherical shape [48]. The effect was that the mask introduction in the irradiated particle surface led to a smaller number of optical vortices near the particle shadow-side surface. The mask 
apodization also increased the effective numerical aperture of the particle-lens, which was equivalent to the increase in the refractive index of the particle material [49-51]. It was also experimentally verified by a scale model using a copper-masked cubic Teflon particle (solid immersion lens) with $2.75 \lambda$ side length under the plane wave radiation with $35 \mathrm{GHz}$ frequency ( $\lambda=8 \mathrm{~mm}$ wavelength) [51]. In this approach, the lateral resolution and the peak intensity enhancement of the produced photonic jet increased by about 36.4 and $36.0 \%$, respectively. The anomalous apodization effect is illustrated in Figure 4.

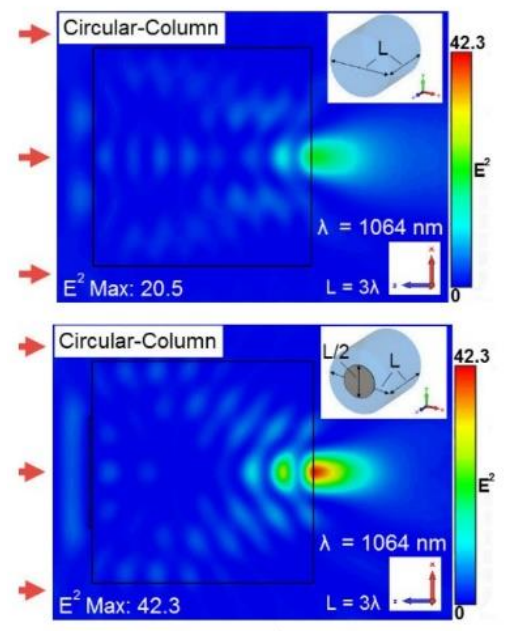

a)

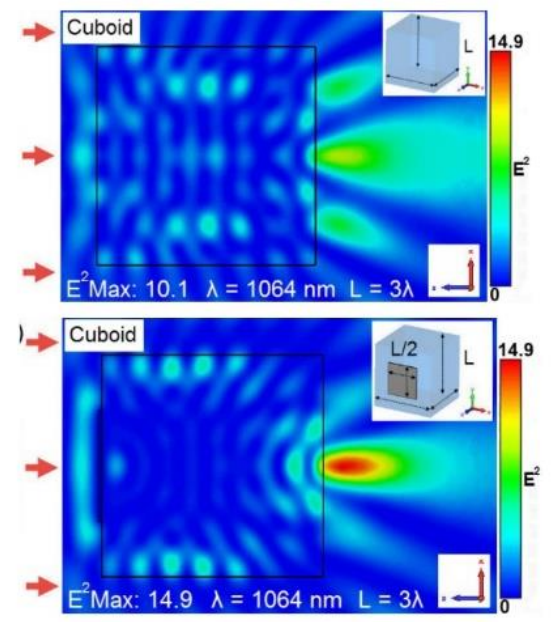

b)

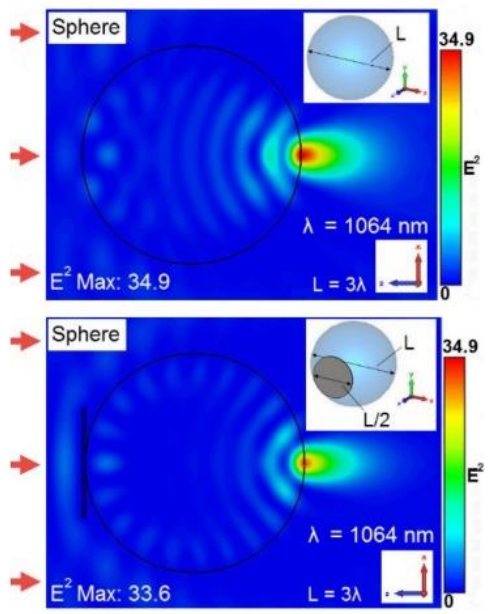

c)

Figure 4. $E^{2}$ field enhancement distribution in $\{x-z\}$ plane for axially illuminated circular-column particle-lens $3 \lambda$ in size (a), cubic (b), and spherical particle (c) without/with the mask apodization.

The mask location, its material and geometry provide the optimization of the field vortex structure (optical flow) inside the dielectric particle and, consequently, the parameter control of the photonic jet. On the other hand, the field vortex configuration near the particle shadow-side surface makes it possible to block the photonic jet formation. In particular, it opens up prospects to study the bound state in the continuum due to strong coupling between Fabry-Perot- and Mie-like modes, and the optical backflow in such structures.

\section{Tangential Electric Field Component Control}

The photonic jet parameter control is possible due to the control for the tangential electric field component along the lateral surface of the dielectric particle using, for example, a metal mask placed along its lateral surfaces [52,53]. Metal screens placed near the lateral surface of the dielectric particle induce a reflected wave, since the respective field component disappears within the contact area. After the wave reflection, the amplitude of the tangential electric field component grows in the direction opposite to that of the wave propagation, and then weakens in the wave propagation direction [52]. These effects lead to a shift of the power flow density concentration region both crosswise and in the wave propagation direction [54]. In particular, it becomes possible to "lock" the field localization region inside a dielectric particle with a refractive index less than the critical when the screen moves along the lateral surface of this particle. We call this effect the "optical syringe". It allows controlling the focal distance of the photonic jet, its length along the radiation propagation direction, and reducing the beam waist near the focus area. On the other hand, a screen placed near one lateral particle surface provides the formation of photonic hooks and angular steering by a photonic jet [53]. Note that the role of the tangential field component is important from a practical point of view, such as, for example, fixing a particle in a microscope holder [55], explaining the effect of increasing focal length. 


\section{Terajets}

The term terajet is introduced by Minin et al. [56] in relation to a photonic jet in the microwave and/or terahertz range. Unlike the optical range, in the terahertz range, the absorption coefficient of the particle material plays a significant role. It can exceed the optical range by several orders of magnitude, and the particle almost always cannot be considered as a weakly absorbed. Note that the first experimental data on the photonic jet effect are presented in [57] for the microwave range.

Dielectric cubic particles have certain advantages over spherical particles [26]: at a linear polarization, the photonic jet generated by the sphere is more elliptical than that generated by cubic particles; the jet length grows for cuboids and shortens for spheres with increasing size of cuboid and the sphere diameter; cuboids generate photonic jets with the minimum particle size of 0.5 wavelength and spherical particles with the minimum diameter of more than a wavelength, etc.

According to [56], the terajet ("teraknife" in a two-dimensional case) moves from inside to outside of the structure with decreasing refractive index of three- and two-dimensional cuboids. This provides a subwavelength size of the beam waist and intensity 10 times higher than that of the illuminating wave. The properties of the dielectric cubic particle as an analog of a flat lens having sizes of the order of a wavelength are retained at the plane wave inclination both on the fundamental frequency and frequency harmonics [58]. Moreover, on a par with the optical range [19], in a terajet field the backscattering is enhanced by $\sim 1.53$ and $\sim 10 \mathrm{~dB}$ for $\mathrm{d}_{1}=0.1 \lambda$ and $\mathrm{d}_{2}=0.25 \lambda$ metal particles, respectively [56]. The use of metamaterials for mesoscale particles provides new effects, for example, the photonic jet birefringence [26]. In this early research [26], we present the results of the photonic jet formation based on 2D photonic crystals $(\mathrm{PhC})$ with the high-index $(n=3.32)$ dielectric cylindrical rods in air host medium. We show that the high-intensity peaks localize in a small volume of the PhCs within the rods, that cannot be predicted by the Bloch-waves theory. Since the photonic jet length in the transverse magnetic (TM) mode is about two times shorter than that of the TM mode, the photonic jet is birenfringent. According to Khodzitsky et al. [59], there are another two types of the heterogeneous metamaterial structure based on photopolymer-perforated cuboid, namely the rectangle with circle holes in homogenious dielectric and the alternating layers. The smallest beam waist of $\sim 0.2 \lambda$ is observed for the TM-polarized wave in polylactic acid photopolymer. It is thus possible to separate photonic jets in space for transverse-electric- and TM-polarized waves [26].

The interesting terajet applications include the gain in sensitivity of terahertz detectors by localized downward radiation in a subwavelength volume [60-62], gain in sensitivity of open microwave resonators [63], the use of terajet generating mesoscale particles as a dielectric small-sized antenna in promising terahertz communication systems, including 5G-6G [64,65], and others.

A mesoparticle introduced in the antinode of the open resonator increases its quality factor, since the terajet reduces the antinode size at the resonator center, providing an extremely low effective mode volume, thereby reducing the diffraction losses at the mirror edges. Please, refer to [63] for detailed discussion.

To increase the efficiency of the optical-to-terahertz conversion and photoresponse [66], the mesoscale dielectric single or array of particles are placed onto the gap between the electrodes [67].

\section{Anomalous Gouy Phase Shift}

The additional information about phase fields can remove restrictions to the problem of super-resolution imaging. The Gouy phase shift [68] was investigated for spherical particles with the size parameters $q=9.34$ [69] and $q=21.4$ [70]. It was shown that the phase shift along the axis of the photonic jet propagation was a combination of the classical Gouy phase shift and the phase shift induced by a refraction on a spherical particle. 
At the same time, for cubic particles with the size parameters of $q=3$, the Gouy phase shift model based on the focused Gaussian beam cannot explain the Gouy phase shift in the photonic jet [71]. Therefore, the formation mechanism of the terajet-based (photonic jet) localized radiation differs from that of the classical lens or spherical particle.

Moreover, in [72] the Gouy asymmetric phase anomaly in a terajet under oblique illumination of a dielectric cube was reported on. This anomaly produced the angle of deviation, not known before, between the normal of the Gouy phase distribution and the direction of the incident beam propagation in the place of the photonic jet formation. This angle of deviation exponentially reduced with the wave propagation to a distance of several wavelengths.

The understanding of such phase anomalies allows localizing the radiation in spatial regions that are smaller than the classical diffraction limit of resolution and can be applied in microscopy, optical trapping and nanoparticle manipulations, interferometry [73].

\section{Waveguide Systems Based on Dielectric Particles Chain}

In 1961, Goubau and Sobel developed the first prototype of the lens waveguide with a set of identical lenses [74,75]. In optics, the light periodic focusing effects were studied in relation to a chain of weakly absorbing spheres $2 \mu \mathrm{m}$ in size $(q>12)$ and the refractive index higher than 1.3. It was shown that these effects were attributed to the so-called photonic modes induced by photonic jets with a period of about a size of two spheres arranged close to each other [76-78]. Interestingly, the temporal pulse broadening at a distance of about four particle diameters was not observed due to photonic modes induced by photonic jets, when using a linearly polarized Gaussian pulse of femtosecond duration for a spherical particle chain with the same size parameter $q$. However, during the same pulse propagation in a solid cylinder made of the same material and of the same diameter, the temporal pulse was broadened approximately by two times.

In our work [79], we propose another type of the waveguide consisting of a chain of three-dimensional mesoscale cubic particles (with the side equaling to the wavelength) separated by an air gap (quasi-periodic dielectric waveguide). The work demonstrates the existence of protonic periodic focusing modes induced by terajets along the particle chain. Additionally, we show that the proposed structure possesses reliable performance and is low-sensitive to the particle material losses (up to the tangent losses of $\sim 0.1$, which is $5 \ldots$ 6 orders higher than in optics) due to the fact that the spatial resolution of terajets does not considerably lower near the shadow-side surface of each cube at a significant loss increase.

Unlike the optical waveguide based on spherical particles, the waveguide based on cubic particles does not require the microsphere contact with each other. The contact between spherical particles can be implemented by microwelding, which, however, decreases the waveguide properties due to microcracks forming after the material melting in the contact area [80,81].

The light periodic focusing effects are also observed for the mesoscale particle chain with $n \sim 1$ refractive index [82]. As can be seen from Figure 5, we have a waveguide with the graded index, i.e., the refractive index gradually lowers from the waveguide center to its edge due to a specific particle shape (sphere, cube) at a constant refractive index inside the particles. Although the downward radiation propagates at the same rate at the waveguide input, the time of reaching its output is different, since the wave propagation velocity at the end is higher than at the center, which leads to the modal dispersion. In fact, this type of the waveguide can be considered as an analog of the waveguide with the gradient (parabolic) refractive index $[83,84]$. 


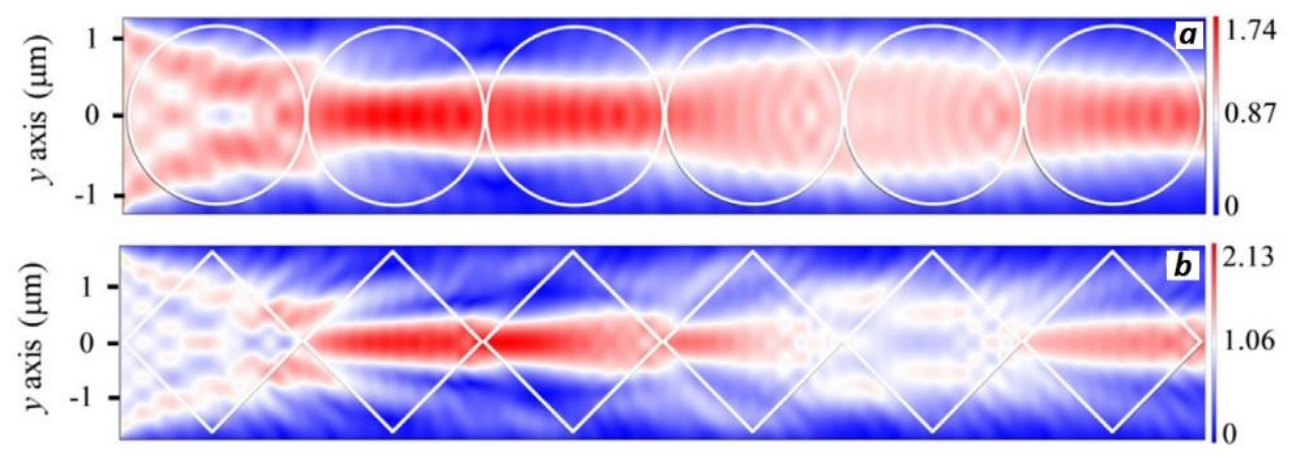

Figure 5. Waveguide with (a) spherical $(n=0.87)$ and $(\mathbf{b})$ cubic $(n=1.06)$ particle chains.

It is interesting to mention the use of mesoscale particles alloyed by optically nonlinear and/or active materials that provides rather interesting optical parametric and laser effects in these structures that can be used to improve the resolution of an isolated particle in the far-field region.

\section{Specular-Reflection Photonic Nanojet}

A specular-reflection photonic nanojet is a type of electromagnetic subwavelength spatial radiation localized in the near-field region, which results from constructive interference of direct and back waves focused by a transparent dielectric mesoscale particle located near the flat reflecting mirror, and is directed toward the wavefront incident $[27,28]$.

For example, the thickness selection of the dielectric particle located on the metal screen provides the formation of the photonic jet with a 1.07 ellipticity, i.e., almost axially symmetric focus at linear polarization. The control for the spatial position of the photonic jet is also possible, until it becomes parallel to the flat surface of the metal screen. This control is performed through a rotation of the whole structure relative to the radiation incidence $[27,85]$. Such a way of the structured field formation is possible for dielectric mesoscale particles of different shapes, including hemispherical [86].

The applicability of Babinet's principle of the additional diffraction structures is also demonstrated in [87] for the photonic jet generation in the reflection mode in the near-field region. Such structures open up possibilities of creating photonic jets with the required and controlled properties, such as focal distance, width, length, maximum field enhancement, and jet ellipticity.

At the same time, the photonic jet formation in the reflected wave allows avoiding several restrictions to the refractive index of the particle material, associated with the photonic jet region in the propagated radiation [5]. According to [86], the photonic jet can form in the opposite direction of the incident wave propagation, in using a nearunity-refractive-index particle on a dielectric substrate with high index contrast or a metal substrate. In this case, the beam width can be less than the diffraction limit. The analysis of the Poynting vector distribution shows [88] that this phenomenon is associated with a multiple circulation of the power flux in and near the particle. It should be noted that the selection of the refractive index contrast between the particle and the substrate can provide the parameter control of the localized radiation area and its position in both reflected and propagated waves. In Figure 6, one can see the electric field intensity distribution at the incident plane wave on the spherical particle with $\sim 1(n=1.077)$ refractive index placed on dielectric substrates with 3.83 and 4.08 refractive indices, respectively. In the first case, we observe a standing wave-modulated photonic jet in the reflection mode and a weak region of localization in the propagated wave. Additionally, in the second case, we see the refractive focusing in the propagated wave and a weak localization in the reflected wave. 


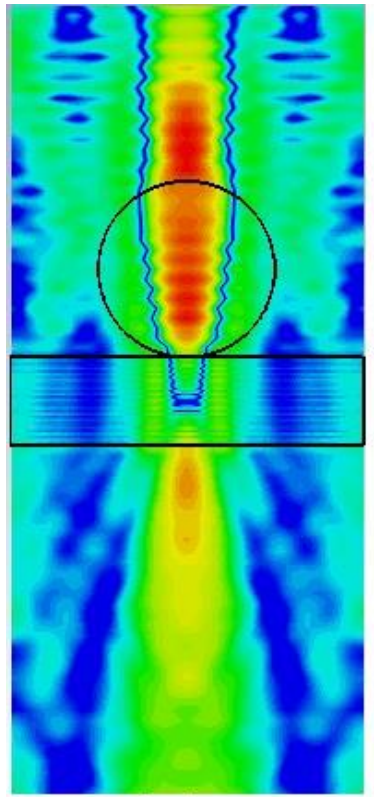

(a)

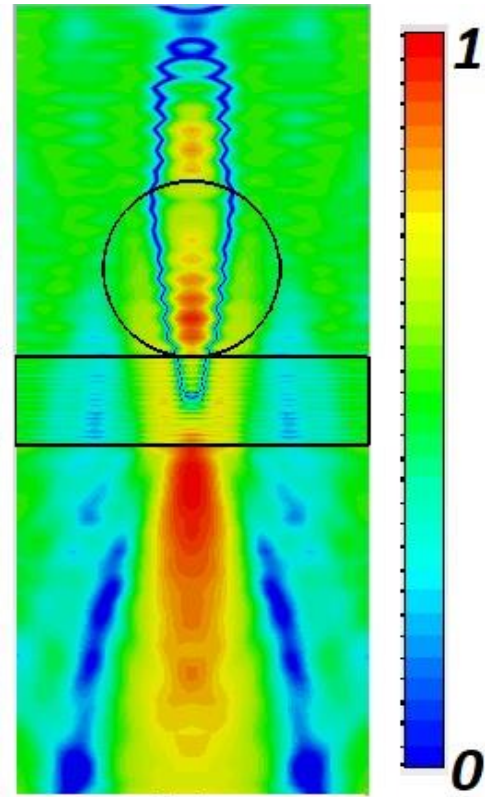

(b)

Figure 6. Generation of photonic jet for spherical particle $(n=1.077)$ positioned on dielectric substates: (a) $n_{1}=3.83$, (b) $n_{1}=4.08$.

Another unique property of photonic jets in the reflection mode is their spatial subwavelength localization and high intensity in using mesoscale particles with a relatively high contrast of the refractive index, when a common photonic jet does not generate $(n>2)$ [89]. In such a configuration, a double focusing of the plane incident wave occurs with the help of a particle locating near the flat metal mirror.

This possibility to overcome the fundamental restriction of the maximum allowable contrast of the refractive index of the particle material is conditioned by the fact that at a specular reflection of radiation followed by the dielectric-particle focusing, large-scale vortices with oncoming circulating optical energy appear in this particle, that contributes to the extreme value of the optical field concentration.

In [89], a physical concept was proposed for the optical tweezer, in which the stability to the Brownian motion of a trapped particle was two times higher than in conventional trappings based on photonic nanojets [90]. The standing wave modulation of the photonic jet could be eliminated, if necessary, due to the interference control [91].

The property control of the spatial propagation of narrow light beams such as divergence and focusing, are the main problems in optics and photonics. Another interesting photonic jet effect is that the dielectric-metal thin rectangular structure can function as a flat focusing mirror $[27,28,92]$. The new type of a planar device without the pre-specified optical axis [92] provides focusing in the near-field region, where the structured reflected beam demonstrates non-diffracted propagation by a distance of the photonic jet length and can significantly enhance the applicability of the structured photonic systems to control the light beam propagation in mesoscale photonic circuits. Note that this type of the near field focusing flat mirror is much simpler than that proposed in $[93,94]$ with a transverse invariance, and is based on other physical principles.

\section{Overcoming the Diffraction Limit and Image Quality Improvement}

According to [95], the whispering gallery mode effect in the microsphere leads to super-resolution imaging up to $\sim 0.25 \lambda$. In [7], it is shown that at a resonance frequency matching the size parameter $q=26.94163$ of the spherical particle, the hotspot size can be about $0.22 \times 0.37 \lambda$ for the linear polarization of the incident field at over 43,000 intensity. The achieved focusing parameters are very sensitive to the size parameter of the spherical 
particle, i.e., its change by $10^{-4}$ reduces the intensity by four orders of magnitude. The similar effect of resonance super-resolution imaging is considered in [96]. It is interesting to note that $0.27 \times 0.6 \lambda$ focal spot for the linear polarization of the illuminating wave can be obtained in an off-resonance frequency mode for a specific configuration of a zone plate [97] with a low level of the side lobes, but at a maximum field intensity, which is 1000 times lower. This is shown in Figure 7.

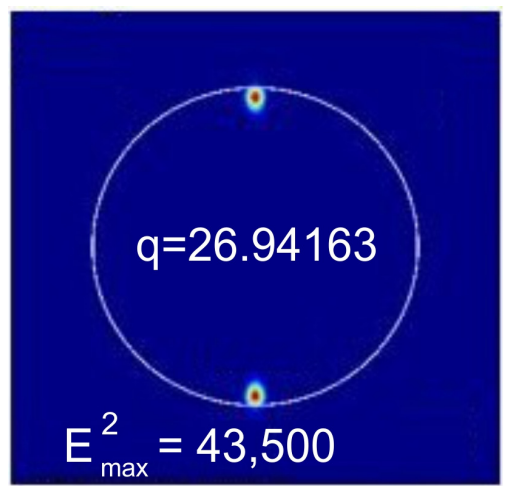

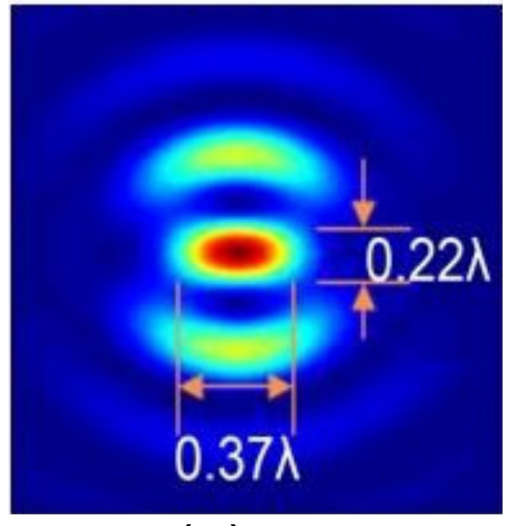

(a)
SIL ZP

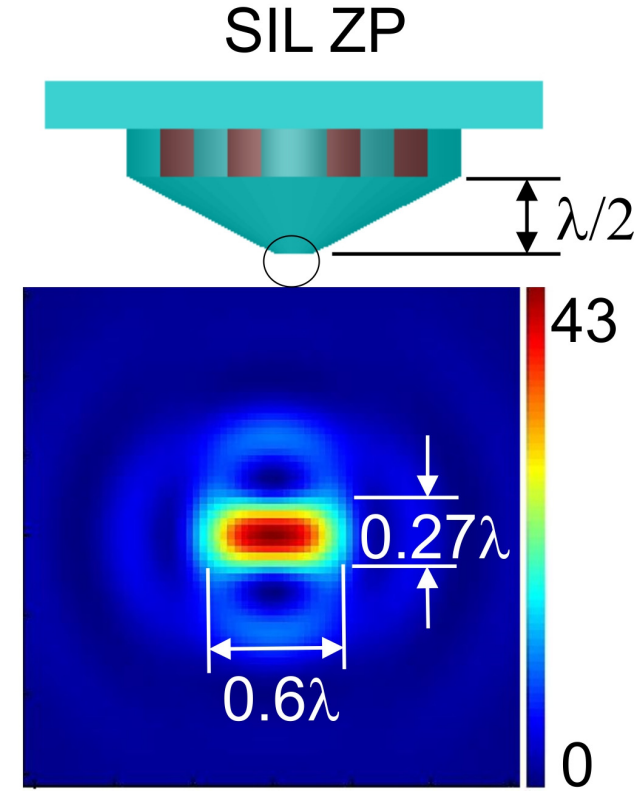

(b)

Figure 7. Formation of localized radiation for spherical particle at resonance frequency (a) and zone plate with immersion cone on off-resonance frequency $(\mathbf{b})$ at linear polarization.

The hotspot generation effect at the poles of spherical particles can be used to detect nanoparticles, including viruses, when their presence in the hotspot region must lead to the resonance frequency shift.

A transfer from common to metamaterials allows dielectric particles with a size of about a wavelength to gain super-resolution, i.e., the beam waist size up to 0.2 wavelength [98], which is rather smaller than the classical diffraction limit. At the same time, the reduction in the beam waist size is also possible due to the use of the gradient particle materials [99]. These effects can assist in solving problems of optical metrology of super-high resolution [100].

In [101] we study a new mechanism of the field localization in mesoscale Janus particles [102] based on truncated spheres or cylinders. It is found that the electric field on a plane surface of such particles has sharp resonances depending on the depth of the removed segment of a sphere or cylinder. These resonances are attributed to the excited whispering gallery modes caused by a truncation of the particle surface. This effect optimized for cylindrical particles allows reaching super-resolution on their plane surface [101].

The physical principles of the truncated spherical lens with focusing without aberrations, also known as the Weierstrass lens, are based on the beam compression formed by the decrease in the refracting angle of the propagated radiation measured on the optical axis. This occurs when the sphere is truncated down to the thickness of $d=R^{*}(l+l / n)$, where 
$R$ is the sphere radius, $d$ is the applanate focus $[103,104]$. However, for low truncation values (small $d$ values), the field redistribution is observed due to the strong excitation of whispering gallery modes and the enhancement of both electric and magnetic fields. Singularity associated with the phase discontinuity on a trace of spherical (cylindrical) surface and plane surface changes Snell's law into laws of reflection and refraction [105]. In accordance with this law, the critical angles of the total internal reflection change, and a critical angle of incidence exists at a certain value of the phase gradient [105]. The latter depends on the thickness of the truncated $d$ element and the refractive index $n$. Figure 8 presents the interference of two evanescent waves. It is found that the effectiveness of excitation by the whispering gallery wave method strongly depends on the truncated $d$ element.
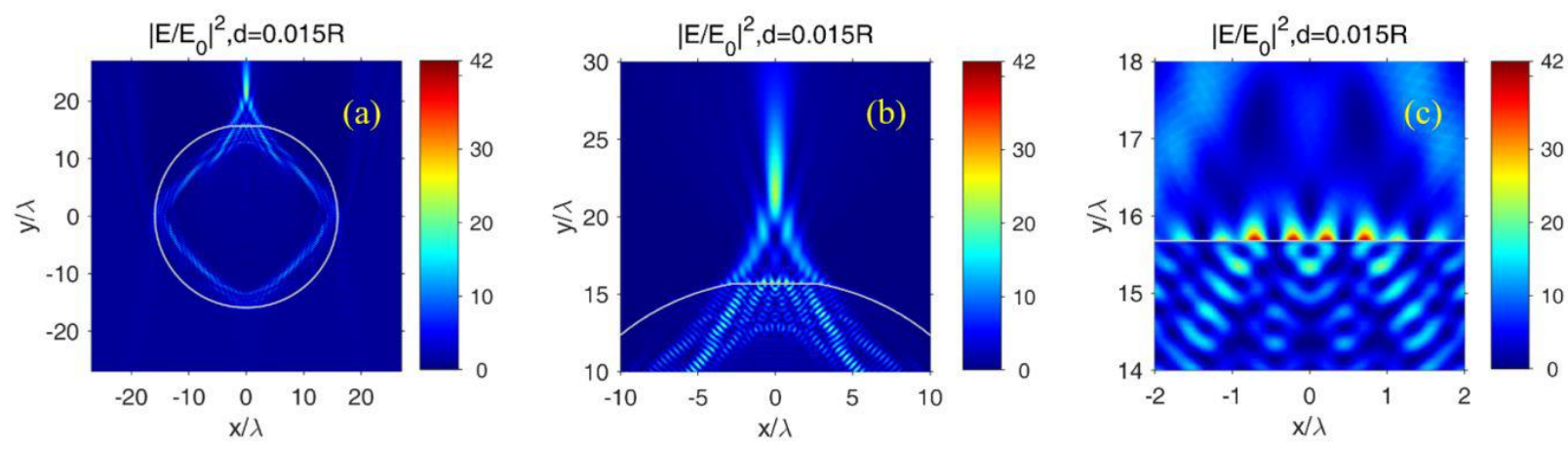

Figure 8. Field intensity distribution for truncated segment resonance and its fragments. Plane front incidents from bottom to top. Adapted from [101].

The use of Janus particles provides the growth in robustness and electric and magnetic field components near the surface of the removed particle element. This effect is resonance in relation to the volume of the removed fraction of the substance and is observed within $q=30 \ldots 90$.

The microsphere introduction in the circuit of a common optical microscope leads to undesired aberrations that lower the image contrast and quality despite the improved localized resolution in the region under the microsphere. Minin et al. [106] demonstrate for the first time that the cubic Teflon dielectric particle with the side equaling a wavelength can be used to improve the spatial resolution of terahertz imaging systems by its placing in the focal area of a lens, independently of the numerical aperture. This approach can be used both in reflecting and transmitting imaging systems [107]. In using $125 \mathrm{GHz}$ frequency in image formation systems, the resolution can be enhanced by 2.2 times, which matches $275 \mathrm{GHz}$. This is especially important in the terahertz range, where the frequency growth of the probing radiation leads to the increase in the medium absorption, and thus cannot be applied. In this case, the image contrast enhancement is 4.4.

The lowest resolution in optical microscopy is limited by the fundamental diffraction limit. Microscopes based on dielectric microparticles are promising tools for overcoming the diffraction limit. However, microparticles have a low image contrast in air, which restricts the application of this method. Using the microparticles that provide the formation of the localized radiation at an angle of the radiation incidence (optical axis), it is possible to increase the image contrast, for example, by photonic hook or jet with the oblique subwavelength illumination in the near field [108]. By analogy with classical optical microscopes $[109,110]$, in the case of the axial formation of the photonic jet, the radiation falls onto an object, and diffracted orders of -1 and +1 are beyond the particle boundaries, while the oblique illumination results in the diffracted order of +1 on the particle, which finally allows increasing the image contrast [111] (see Figure 9). 

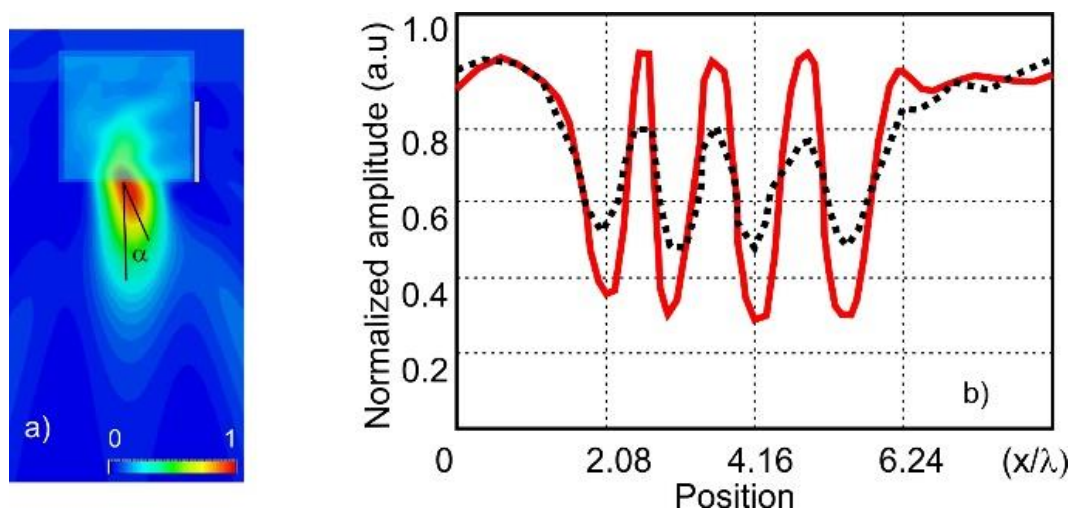

Figure 9. Formation of photonic hook for cubic particle with lateral mask (a); image contrast of test object in photonic jet radiation (dotted curve) and photonic let (solid curve) (b).

\section{Nanostructured Particles with Refractive Index near 2}

As mentioned above, the transverse size of the localized field of a dielectric mesoscale particle is usually larger than $\lambda / 3$ at the refractive index below the critical. For the first time, in [112,113], it is shown that the region of the localized electromagnetic field forming near the shadow surface of the dielectric mesoscale spherical or cubic particle with the refractive index of two of its material, can be substantially reduced after the nanohole introduction in its shadow surface, which improves the spatial resolution up to $\lambda / 100$. Such an introduction allows compressing the field localization characteristic to the photonic jet, down to the nanohole size thanks to the permittivity contrast between the particle and nanohole; the minimum beam waist is determined by the nanohole size rather than the wavelength. At the same time, the analysis of the optical response of a particle based on the multipole decomposition shows that the nanohole weakly affects the optical response of the particle in the nonresonance mode.

It is known that a circulating energy flow arises around a phase singularity containing a reverse flow [2-5]. Thus, the localized field region formed by a mesoscale particle with the critical or slightly higher than the critical refractive index contrast $[89,114]$ and having a nano-hole on the axis, can be used to separate regions with forward and backward energy flows [115]. Such nanostructuring of the particle's shadow-side surface with the refractive index of two provides the development of concepts of the optical vacuum cleaner [113] and optical magnet [115] for the nanoparticle manipulation, and optical modulation based on wavelength-scaled dielectric particle with the graphene monolayer [114].

A more complex nanostructuring of the particle shadow-side surface with the refractive index less than two, based on the diffraction structures for optical manipulation was considered in [116].

\section{Structured Fields in the Form of Photonic Hooks and Loops}

Recently, a new family of near-field localized curved light beams was discovered, known as photon hooks, which differ from Airy beams $[117,118]$. The term photonic hook and its concept were introduced in [26,119]. Photonic hooks are unique, because their curvature radius is much smaller than the wavelength, which means that such hooks can be maximally accelerated, and the side lobes do not fit the shape of the main beam and are not curved. Moreover, in photonic hooks, there is a flex point, where the beam changes the direction of propagation. Photonic hooks can be observed near the shadow-side surface of the Janus particles, and they are free form other restrictions. Airy beams have no such properties [118].

In the simplest case, Janus particles in the form of a dielectric cube with a prism [26,119-121], and the interference induced by the different particle thickness along the polarization direction, lead to the additional singular points near the shadow-side surface of the asymmetric particle. In the latter, the time of the total phase of the optical wave oscillation changes, 
resulting in the beam curvature. Moreover, a change in the incident radiation wavelength or/and polarization leads to the nanovortex spatial redistribution, thereby changing the photonic hook spatial position and structure.

There are currently several approaches to the formation of the curved beams such as photonic hooks, systemized in [122]. The approaches include asymmetry of the particle shape at a constant refractive index $[123,124]$, symmetric particles with the refractive index asymmetry [125-127], symmetric uniform particle with the asymmetric illuminating wave $[128,129]$, symmetric particles in inhomogeneous (e.g., with discrete refractive index distribution) medium, a system of mesoscale scatters [130], and a combination of these approaches. These approaches can generate localized structured beams of various configurations. In Figure 10, one can see the field configurations for a photonic hook and optical capsule, and an optical trapping based on a structured cylindrical particle (cylinder with $\pi / 2$-cut segment). In the latter case, the cylindrical particle is irradiated by a spatial-limited wave, whereas the cut segment in the shadow-side surface provides the formation of two photonic jets, whose interference forms the region of a standing wave of higher intensity (a photonic jet from the upper half of the cylinder falls on the flat surface of the cut segment and is reflected from it). As shown in Figure 10b, the elongated shape of the bottle-like region of such 'nanophotonic tweezers' and exciting new class of 'on-chip' optical traps allow using this trapping for anisotropic nanoobjects, which is rather difficult in classical configurations. These concepts can also be developed to obtain near-field beams of the photonic loop type [125].
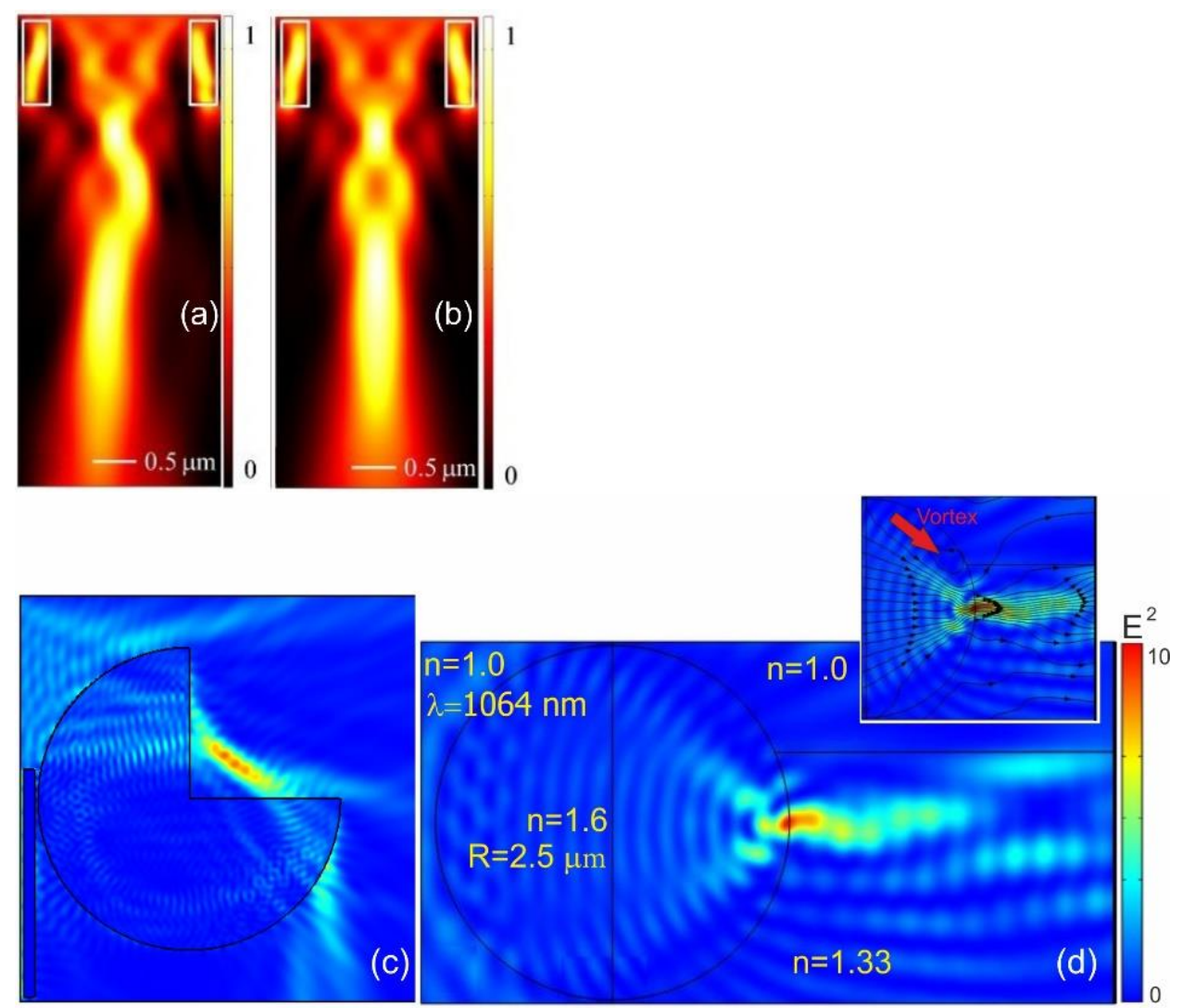

Figure 10. Structured fields in the form of photonic hook (a) and bottle-like region of localized field (b) based on a pair of dielectric rods. (c) optical trapping in standing wave region, (d) concept of the structured light in the form of photonic hook in step-index medium. 
The structured light in the step-index medium such as photonic hook is shown in Figure 10b (Comsol simulation model created by Yu. Geints). As can be seen from this figure, the mesoparticle is located in a homogeneous medium (air). In the shadow-side surface an inhomogeneous medium is present, which is, for example, a two-layer step-index air-water structure. The thickness of water and air layers determines the configuration of the localized structured field. In this case, the water/air interface playes a significant role in the structured field formation, since the vortex flow occurs inside the particle at this interface. Note that the localized beam is modulated by a standing wave due to the gradient contrast of the refractive index of the two media.

The photonic hook concept suggests the control for the particle motion to manipulate and sort cells on the laboratory on-a-chip platform. A unique property of the photonic hook is the subwavelength beam curvature, that can be used to transport nanoparticles outside the obstacle [119]. In the approximation of a Rayleigh particle, it is shown that it can be moved round the dielectric plate-obstacle [131]. At the same time, a simple change in polarization can switch the spatial orientation of the field localization $[128,129]$. Some of in vitro biomedical applications of this concept include the cell motion over the curved trajectory for their successive differentiation [132], and nanoobject transportation to the intercellular or intracellular media by a curved trajectory for nanodrug delivery via the central nervous system barriers [133], laser surgery [134], microfluid devices, surface nanostructuring, new photonic devices [135], and many others.

The near-field control will be more and more important in the integrated photonics. For example, prospects are opening up for constructing devices for spectral and spatial separation of radiation incident on a particle, using the photonic hook and jet phenomena. In this regard, structured beams of the photonic jet and photonic hook type, can be a bridge to connect the photonic and physical worlds. It is interesting to note that the properties of pyramidal meso-particles $[27,28,136,137]$ probably disclose the nature of ancient pyramids [138-140].

\section{Low-Dimensional Systems. Plasmonic Jets and Hooks}

The study of the properties of low-dimensional systems occupies one of the central positions in photonics. Plasmonic structures are intensively studied due to their ability to localize the light at subwavelength scales [141,142]. Surface plasmonic waves are twodimensional waves, whose field components exponentially decay as they move away from the metal-dielectric interface.

Fundamentally, during the transition from three-dimensional to two-dimensional space, the quantum nature of the matter manifests itself. Therefore, there are new possibilities for controlling the interaction between the material and light.

The possibility of formation of the photonic jet analog for plasmonic waves based on a dielectric disk placed on a metal film was first theoretically shown in [143]. Later, the formation of a plasmonic jet based on a dielectric cuboid of silicon nitride $\left(\mathrm{Si}_{3} \mathrm{~N}_{4}\right)$ was investigated in [144] using the solution of Maxwell's equations. The idea of a waveguide based on the cubic particle chains [79] provided the increase in the propagation length of the localized plasmonic wave [145].

However, the plasmonic jet effect based on a dielectric cube on a gold film was first confirmed experimentally in [146]. The plasmonic jet effect demonstrated exciting potential applications in integrated and near-field optics for the plasmonic wave control. Such a new and simple platform could provide a new path for plasmonics, high-resolution imaging, biophotonics, optical data storage, and future applications from nanoscale light control to on-chip photonic devices [147]. 
The same relates to the plasmonic hook. The plasmonic hook effect was theoretically justified in [148], and just recently it was first confirmed experimentally [149]. The singular points inside the Janus particle induce the curvatures of the plasmonic beam. It is important to note that the plasmonic hook demonstrated the smallest beam curvature ever recorded for plasmonic waves. It could be noted that using the idea of a surface plasmon linear waveguide [145] and results of the works [148,149] it is possible to construct curved surface plasmon waveguides using an array of Janus particles arranged along a curved path.

The experimental verification of these effects opens up new possibilities for controlling the interaction between the light and matter at the nano-level and can greatly expand the available tools for new light manipulations [150].

\section{Acoustic Jets and Hooks}

In the optical range, two parameters are usually sufficient to characterize photonic jets: the Mie size parameter and the refractive index contrast. In the terahertz and microwave ranges, a parameter characterizing losses in the particle material is added. In the acoustic range, another parameter is added; all materials, as a rule, are anisotropic due to the presence of two sound velocities in the solid particle material: longitudinal and transverse (shear wave).

The term acoustic jet was introduced by Minin I.V. and Minin O.V. in [151] by analogy with Maxwell's equations and continuum mechanics for the linear mode. Works [152,153] experimentally demonstrated the acoustic jet generation with a minimum beam waist size of the acoustic jet of about half the wavelength for spherical and cylindrical particles. A significant influence on the acoustic jet formation exerted by the ratio between the axial and transverse sound velocities in the particle material was shown in [154]. Acoustic jets based on metamaterial particles were investigated in [151,155]. The effect of anomalous apodization for acoustic jets in both gas and liquid was confirmed in $[156,157]$. It should be noted that according to the in-depth study, a simple analogy between acoustics and optics [151] was valid only in the first approximation, since in acoustics, one more additional free parameter — the particle density—must be used [122].

According to Figure 11, in the acoustic jet formation, just as in optics, an essential role is given to the system of acoustic vortices both inside the particle material and near its shadow surface.
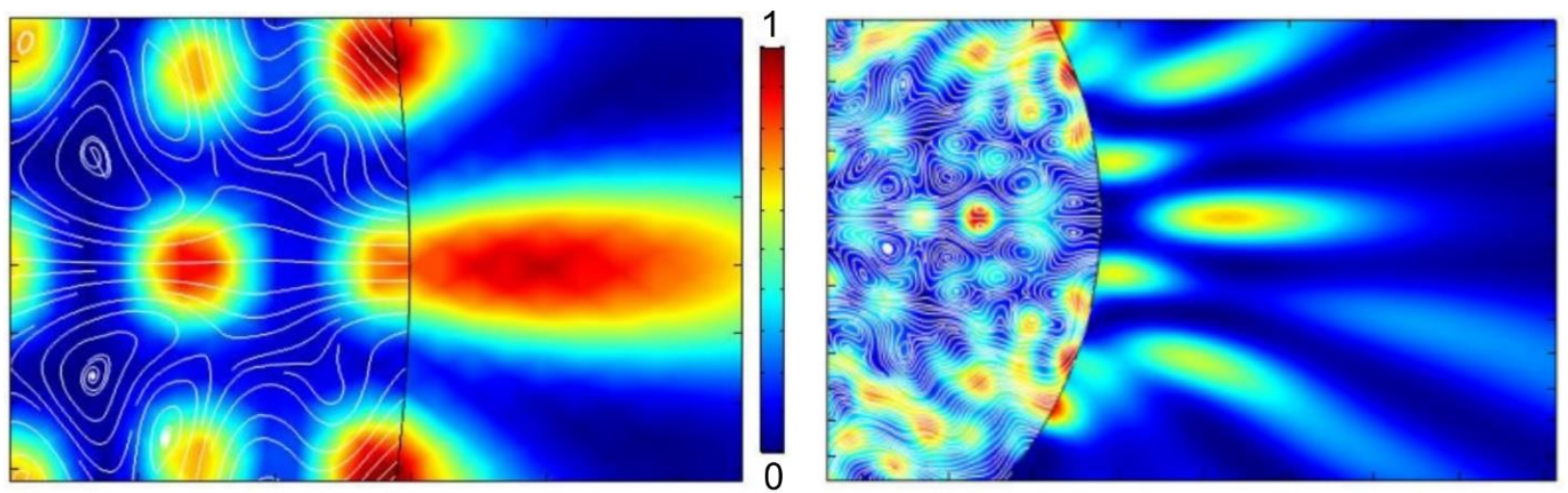

Figure 11. Vortices in the Rexolite (density: $1049 \mathrm{~kg} / \mathrm{m}^{3}$, axial sound velocity: $2337 \mathrm{~m} / \mathrm{s}$, transverse sound velocity: $1157 \mathrm{~m} / \mathrm{s}$ ) particle and hotspot generation in off-resonance (left) and near-resonance (right) modes.

In addition, the optical super-resonance effect was also observed in acoustics [156,157] as well as the extremely high acoustic wave intensity presented in [158,159] (see Figure 12). Near the resonance frequency, the resolution in the hotspot region is about $(0.2-0.23) \lambda$. 

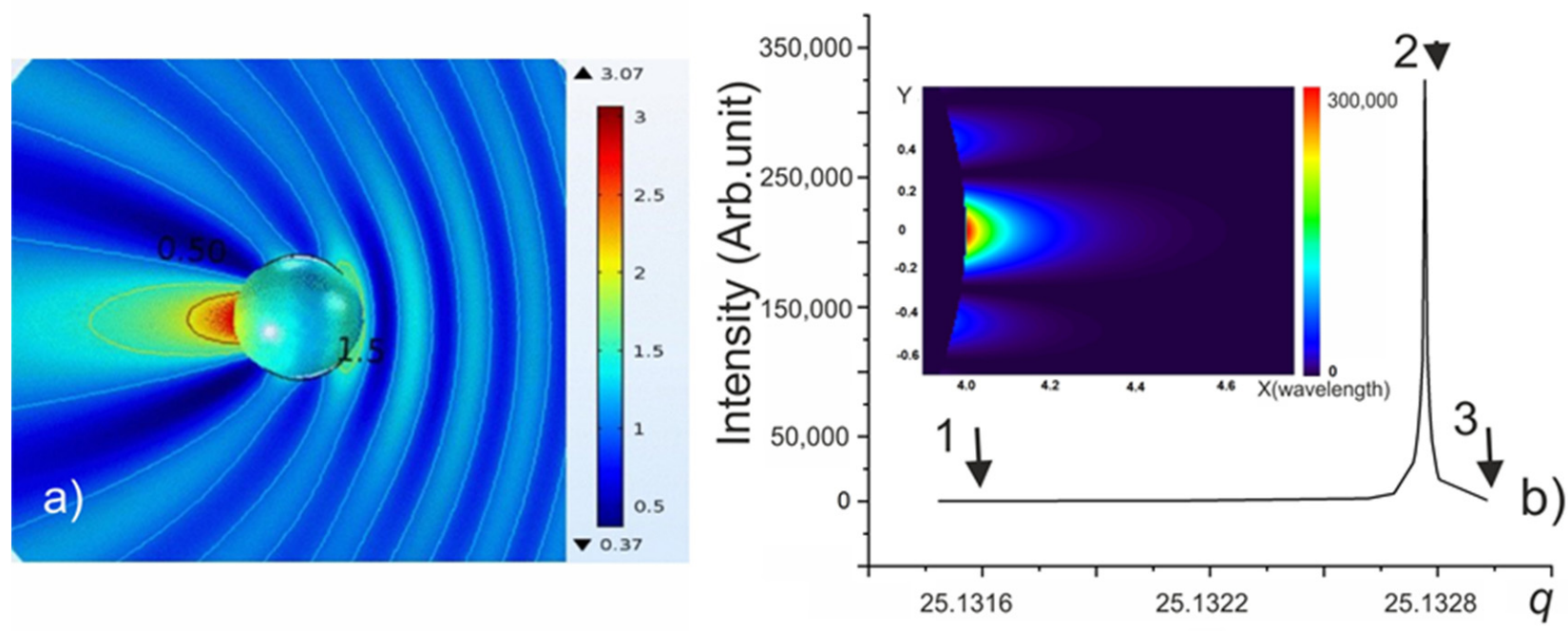

Figure 12. Acoustic jet in non-resonant mode for particle with $q \sim 2 \pi$ (a) and resonance scattering of acoustic wave on a spherical Rexolite particle located in water depending on Mie parameter (b). Inset: hotspot structure on the particle shadow-side surface at the resonant frequency. Maximum intensity at points 1-3 are: 325 (1), 325,000 (2), 1000 (3) in units of the acoustic wave intensity incident on the particle.

The photonic hook concept was extended to acoustic waves and experimentally confirmed in [160]. This curved acoustic beam was generated by using a Rexolite particle immersed in water with a trapezoidal rectangular prism, on which a plane ultrasonic wave with a $250 \mathrm{kHz}$ frequency fell. The same results were obtained later in [161]. In [162], acoustic jets and hooks in water were obtained using a mesoscale $(q<30)$ ABS plastic cylinder fabricated on a 3D printer. It was shown that in the resonance mode, the excitation of whispering gallery modes resulted in the acoustic jet diameters less than the diffraction limit. In the off-resonance mode, the cubic phononic crystal was placed inside the cylinder, and both acoustic jets and acoustic hooks were formed depending on its orientation relative to the wave incidence direction. Later, a particle consisting of a hemispherical Rexolite cylinder and a hemispherical photonic crystal (two different materials with different effective refractive indexes were used) forming an acoustic hook was considered in [163].

The structured acoustic beams such as jets and hooks can also find applications in microscopy, medical imaging [164], monitoring of various structural states [165], energy harvesting and localization [166].

\section{Conclusions}

The electromagnetic field localization and manipulation in the small volume up to a deep subwavelength, are fundamental for research into dielectric mesophotonics. This paper is focused on novel strategies employed to manipulate subwavelength structured optical, acoustic and in-plane plasmonic fields, enhance localized magnetic and electric fields, and photonic wavelength-scaled particle-based waveguide.

Three- and two-dimensional dielectric mesostructures is a relatively new research field in photonics and in-plane plasmonics (and even in acoustics), which is based on the physics of relative low-index wavelength-scaled dielectric particles with the Mie size parameter of $q \sim 10$ enhanced by the interference effects between the different field components generated in or/and near the particle shadow-side surface. Different possibilities are therefore provided for both non-resonance and resonance modes. An additional key point is that the dielectric mesoscale particles can be fabricated from well-known dielectric materials of a common shape such as spherical, cylindrical or cubic, that can naturally be made by standard processing. On the other hand, one of the mesoparticle limitations is that the localized field is limited to the particle size of the order of $2 R>\lambda$.

Optical singularities, in particular vortices and their acoustic counterparts, are intriguing deep physics phenomena that allow for interesting applications. For example, 
the angular and linear momentum of photons in structured fields can be used for optical capture and manipulation of nanoparticles and biological micro-objects $[167,168]$.

With auxiliary mesoscale dielectric particles of an arbitrary 3D shape, it was possible to generate structured fields, which allowed increasing the flexibility and creating more varied kinds of optical manipulation with unusual properties of the optical forces beyond trapping.

Mesoparticles can support high-order Fano super-resonances, which provide the giant magnetic field and hot spot generation via high-order Fano resonances, that can be used in the super-resolution microscopy. The generation of giant electric and magnetic fields in dielectric mesoscale particles is comparable to the fields generated in plasmonic structures and can be used to destroy viruses [169], optical capture of particles with a high magnetic polarizability [170].

The photothermal therapy method proposed in [171], is used to deliver a heat dose to a given area of the living tissue via metal nanoparticles lethal for cancer cells. These nanoparticles are introduced into the body and bind to cancers. However, the absorption peak of the traditional gold spherical nanoparticles is in the visible range. In order to shift the plasmon resonance toward the near-infrared range and trap nanoparticles by the target, MXene $\mathrm{Ti}_{3} \mathrm{C}_{2}$ nanoparticles are used in the photonic hook [124].

A separate major direction is associated with the use of mesoscale particles in microscopy $[38,39,106,172]$. In the authors' opinion, the creation of mesoparticle chains with the required properties using the phase-matching mechanism that relies on the coupling of random quasi-phase-matching with the Mie resonance of the entirely disordered mesostructure is promising [173]. The generation of hot spots, having giant values of the local wavenumber vectors, by use a superoscillation effects $[9,10]$ is extremely promising not only for mesoscale photonics and superresolution imaging, but also for diffractive optics [174], whose research was begun back in 1990 [175].

The use of Janus particles, the simplest of which is a hemisphere (half-cylinder) [176], with various types of asymmetry mentioned above, allows for the flexible control of structured fields depending on the application. The particle in the resonant fundamental mode (modes with the smallest volume corresponding to $|\mathrm{m}|=1$, and $\mathrm{s}=1$ ) allows using it as a cloak structure [177].

We believe that new insights will be delivered through the development of related fields and expect that the future research into dielectric mesophotonics will offer new opportunities leading to novel unusual applications of photonics. In particular, the effects considered above make it possible to explain the anomalous crater during laser nanostructuring of the surface with the help of particles with parameter $\mathrm{q} 2 \pi$ [178]. A combination of the mesoscale particle concept with materials with nonlinear properties will be promising and attractive. The obtained results show the potential for wavelength-scaled dielectric particles to manipulate radiation beyond the limitations of the geometrical macro optics [179]. Effects negligible at the macro optics are important at the mesolevel and vice versa. The wavelength-scaled dielectric particle potential for various applications still remains unstudied. In general, new physical effects and applications indicate to the development of a new promising direction in optics, acoustics, and in-plane plasmonics.

Author Contributions: Conceptualization, I.V.M. and O.V.M.; methodology, I.V.M. and O.V.M.; writing-original draft preparation, I.V.M. and O.V.M. All authors have read and agreed to the published version of the manuscript.

Funding: This research received no external funding.

Institutional Review Board Statement: Not applicable.

Informed Consent Statement: Not applicable.

Data Availability Statement: Not applicable. 
Acknowledgments: The authors are grateful to all their co-authors on joint previously published articles for their participation in research at different stages. The work was carried out within the framework of the Tomsk Polytechnic University Development Program, Russia.

Conflicts of Interest: The authors declare no conflict of interest.

\section{References}

1. Mie, G. Contributions to the optics of turbid media, particularly of colloidal metal solutions. Ann. Phys. 1908, 25, 377-445. [CrossRef]

2. Luk'yanchuk, B.; Ternovsky, V. Light scattering by a thin wire with a surface-plasmon resonance: Bifurcations of the Poynting vector field. Phys. Rev. B 2006, 73, 235432. [CrossRef]

3. Bashevoy, M.; Fedotov, V.; Zheludev, N. Optical whirlpool on an absorbing metallic nanoparticle. Opt. Express 2005, 13, 8372. [CrossRef]

4. Luk'yanchuk, B.; Miroshnichenko, A.; Kivshar, Y. Fano resonances and topological optics: An interplay of far- and near-field interference phenomena. J. Opt. 2013, 15, 73001. [CrossRef]

5. Luk'yanchuk, B.; Paniagua-Domınguez, R.; Minin, I.V.; Minin, O.V.; Wang, Z. Refractive index less than two: Photonic nanojets yesterday, today and tomorrow. Opt. Mater. Express 2017, 7, 1820-1847. [CrossRef]

6. Yue, L.; Yan, B.; Monks, J.; Dhama, R.; Jiang, C.; Minin, O.V.; Minin, I.V.; Wang, Z. Full three-dimensional Poynting vector flow analysis of great field-intensity enhancement in specifically sized spherical-particles. Sci. Rep. 2019, 9, 20224. [CrossRef] [PubMed]

7. Wang, Z.; Luk'yanchuk, B.; Yue, L.; Yan, B.; Monks, J.; Dhama, R.; Minin, O.V.; Minin, I.V.; Huang, S.; Fedyanin, A. High order Fano resonances and giant magnetic fields in dielectric microspheres. Sci. Rep. 2019, 9, 20293. [CrossRef]

8. Yue, L.; Wang, Z.; Yan, B.; Monks, J.; Joya, Y.; Dhama, R.; Minin, O.V.; Minin, I.V. Super-Enhancement Focusing of Teflon Spheres. Ann. Phys. 2020, 532, 2000373. [CrossRef]

9. Berry, M.V. Superoscillations and leaky spectra. J. Phys. A Math. Theor. 2018, 52, 15202. [CrossRef]

10. Zheludev, N.I.; Yuan, G.H. Optical superoscillation technologies beyond the diffraction limit. Nat. Rev. Phys. 2021. [CrossRef]

11. Sakharov, A.D. Magnetoimplosive generators. Sov. Phys. Uspekhi. 1966, 9, 294-299. [CrossRef]

12. Minin, V.F.; Minin, I.V.; Minin, O.V. Calculation Experiment Technology. In Computational Fluid Dynamics; InTechOpen: London, UK, 2011. [CrossRef]

13. Chen, Z.; Taflove, A.; Backman, V. Photonic nanojet enhancement of backscattering of light by nanoparticles: A potential novel visible-light ultramicroscopy technique. Opt. Express 2004, 12, 1214-1220. [CrossRef]

14. Benincasa, D.S.; Barber, P.W.; Zhang, J.-Z.; Hsieh, W.-F.; Chang, R.K. Spatial distribution of the internal and near-field intensities of large cylindrical and spherical scatterers. Appl. Opt. 1987, 26, 1348-1356. [CrossRef]

15. Luk'yanchuk, B.; Zheng, Y.; Lu, Y. Laser cleaning of solid surface: Optical resonance and near-field effects. Proc. SPIE 2000, 4065, 576-587.

16. Lu, Y.; Zhang, L.; Song, W.; Zheng, Y.; Luk'yanchuk, B. Laser writing of a subwavelength structure on silicon (100) surfaces with particle-enhanced optical irradiation. JETP Lett. 2000, 72, 457-459. [CrossRef]

17. Mosbacher, M.; Münzer, H.; Zimmermann, J.; Solis, J.; Boneberg, J.; Leiderer, P. Optical field enhancement effects in laser-assisted particle removal. Appl. Phys. A 2001, 72, 41-44.

18. Wang, J.; Gouesbet, G.; Gréhan, G.; Han, Y.; Saengkaew, S. Morphology-dependent resonances in an eccentrically layered sphere illuminated by a tightly focused off-axis Gaussian beam: Parallel and perpendicular beam incidence. J. Opt. Soc. Am. A 2011, 28, 1849. [CrossRef]

19. Li, X.; Chen, Z.; Taflove, A.; Backman, V. Optical analysis of nanoparticles via enhanced backscattering facilitated by 3-D photonic nanojets. Opt. Express 2005, 13, 526. [CrossRef]

20. Yousefi, M.; Scharf, T.; Rossi, M. Photonic nanojet generation under converging and diverging beams. J. Opt. Soc. Am. B 2021, 38, 317-326. [CrossRef]

21. Wang, H.; Hao, J.; Zhang, B.; Han, C.; Zhao, C.; Shen, Z.; Xu, J.; Ding, J. Donut-like photonic nanojet with reverse energy flow. Chin. Opt. Lett. 2021, 19, 102602. [CrossRef]

22. Lecler, C.; Takakura, Y.; Meyrueis, P. Properties of a three-dimensional photonic jet. Opt. Lett. 2005, 30, 2641-2643. [CrossRef] [PubMed]

23. Minin, I.V.; Minin, O.V.; Gagnon, N.; Petosa, A. FDTD Analysis of a Flat Diffractive Optics with Sub-Reyleigh Limit Resolution in $\mathrm{MM} / \mathrm{THz}$ Waveband. In Proceedings of the 2006 Joint 31st International Conference on Infrared Millimeter Waves and 14th International Conference on Teraherz Electronics, Shanghai, China, 18-22 September 2006; p. 170.

24. Minin, I.V.; Minin, O.V. Experimental verification 3D subwavelength resolution beyond the diffraction limit with zone plate in millimeter wave. Microw. Opt. Technol. Lett. 2014, 56, 2436-2439. [CrossRef]

25. Liu, C.; Kaiser, T.; Lange, S.; Schweiger, G. Structural resonances in a dielectric sphere illuminated by an evanescent wave. Opt. Commun. 1995, 117, 521-531. [CrossRef]

26. Minin, I.V.; Minin, O.V. Diffractive Optics and Nanophotonics: Resolution Below the Diffraction Limit; Springer: Berlin, Germany, 2016.

27. Minin, I.V.; Minin, O.V. Photonics of isolated dielectric particles of arbitrary 3D shape-A new direction of optical information technologies. Vestnik NSU 2014, 12, 59-70. 
28. Minin, I.V.; Minin, O.V.; Geints, Y. Localized EM and photonic jets from non-spherical and non-symmetrical dielectric mesoscale objects: Brief review. Ann. Phys. 2015, 527, 491-502. [CrossRef]

29. Cui, X.; Erni, D.; Hafner, C. Optical forces on metallic nanoparticles induced by a photonic nanojet. Opt. Express 2008, 16, 13560. [CrossRef] [PubMed]

30. Li, Y.; Xin, H.; Liu, X.; Zhang, Y.; Lei, H.; Li, B. Trapping and Detection of Nanoparticles and Cells Using a Parallel Photonic Nanojet Array. ACS Nano 2016, 10, 5800. [CrossRef] [PubMed]

31. Shakhov, A.; Astafiev, A.; Nadtochenko, V. Microparticle manipulation using femtosecond photonic nanojet-assisted laser cavitation. Opt. Lett. 2018, 43, 1858. [CrossRef]

32. Surdo, S.; Duocastella, M.; Diaspro, A. Nanopatterning with Photonic Nanojets: Review and Perspectives in Biomedical Research. Micromachines 2021, 12, 256. [CrossRef]

33. Lu, D.; Pedroni, M.; Labrador-Paez, L.; Marques, M.; Jaque, D.; Haro-Gonzalez, P. Nanojet Trapping of a Single Sub-10 nm Upconverting Nanoparticle in the Full Liquid Water Temperature Range. Nano Micro Small 2021, 17, 2006764. [CrossRef] [PubMed]

34. Killian, J.; Ye, F.; Wang, M. Optical Tweezers: A Force to Be Reckoned With. Cell 2018, 175, 1445-1448. [CrossRef]

35. Shan, X.; Wang, F.; Wang, D.; Wen, S.; Chen, C.; Di, X.; Nie, P.; Liao, J.; Liu, Y.; Ding, L.; et al. Optical tweezers beyond refractive index mismatch using highly doped upconversion nanoparticles. Nat. Nanotechnol. 2021, 16, 531-537. [CrossRef]

36. Zhang, W.; Lei, H. Fluorescence enhancement based on cooperative effects of a photonic nanojet and plasmon resonance. Nanoscale 2020, 12, 6596-6602. [CrossRef]

37. Bratchenko, L.; Bratchenko, I.; Khristoforova, Y.; Artemyev, D.; Konovalova, D.; Lebedev, P.; Zakharov, V. Raman spectroscopy of human skin for kidney failure detection. J. Biophotonics 2020, 14, e202000360. [CrossRef]

38. Hüser, L.; Lehmann, P. Microsphere-assisted interference microscopy for resolution enhancement. tm Tech. Mess. 2021, 88, 311-318. [CrossRef]

39. Li, P.; Li, G.; Yu, H.; Wang, F.; Liu, L.; Li, W. Advances in Dielectric Microspherical Lens Nanoscopy: Label-Free Superresolution Imaging. IEEE Nanotechnol. Mag. 2021, 15, 38. [CrossRef]

40. Kuznetsov, A.; Miroshnichenko, A.; Fu, Y.; Zhang, J.; Luk'yanchuk, B. Magnetic light. Sci. Rep. 2012, 2, 492. [CrossRef] [PubMed]

41. Albella, P.; Poyli, M.; Schmidt, M.; Maier, S.; Moreno, F.; Saénz, J.; Aizpurua, J. Low-Loss Electric and Magnetic Field-Enhanced Spectroscopy with Subwavelength Silicon Dimers. J. Phys. Chem. C 2013, 117, 13573-13584. [CrossRef]

42. Bakker, R.; Permyakov, D.; Yu, Y.; Markovich, D.; Paniagua-Domínguez, R.; Gonzaga, L.; Samusev, A.; Kivshar, Y.; Luk'yanchuk, B.; Kuznetsov, A. Magnetic and electric hotspots with silicon nanodimers. Nano Lett. 2015, 15, 2137-2142. [CrossRef]

43. Li, C.; Kattawar, G.; Zhai, P. Electric and magnetic energy density distributions inside and outside dielectric particles illuminated by a plane electromagnetic wave. Opt. Express 2005, 13, 4554. [CrossRef]

44. Baranov, D.; Savelev, R.; Li, S.; Krasnok, A.; Alu, A. Modifying magnetic dipole spontaneous emission with nanophotonic structures. Laser Photonics Rev. 2017, 11, 1600268. [CrossRef]

45. Born, M.; Wolf, E. Principles of Optics, 7th ed.; Cambridge University Press: Cambridge, UK, 1999.

46. Yan, B.; Yue, L.; Wang, Z. Engineering near-field focusing of a microsphere lens with pupil masks. Opt. Commun. 2016, 370, 140. [CrossRef]

47. Wu, M.; Chen, R.; Soh, J.; Shen, Y.; Jiao, L.; Wu, J.; Chen, X.; Ji, R.; Hong, M. Super-focusing of center-covered engineered microsphere. Sci. Rep. 2016, 6, 31637. [CrossRef] [PubMed]

48. Minin, I.V.; Minin, O.V. Patent of Russia 21, N 153686, 2015.

49. Yue, L.; Yan, B.; Monks, J.; Dhama, R.; Wang, Z.; Minin, O.V.; Minin, I.V. V. Intensity-Enhanced Apodization Effect on an Axially Illuminated Circular-Column Particle-Lens. Ann. Phys. 2017, 530, 1700384. [CrossRef]

50. Yue, L.; Yan, B.; Monks, J.; Wang, Z.; Tung, N.; Lam, V.; Minin, O.V.; Minin, I.V. Production of photonic nanojets by using pupil-masked 3D dielectric cuboid. J. Phys. D Appl. Phys. 2017, 50, 175102. [CrossRef]

51. Yue, L.; Yan, B.; Monks, J.; Wang, Z.; Tung, N.; Lam, V.; Minin, O.V.; Minin, I.V. A millimetre-wave cuboid solid immersion lens with intensity-enhanced amplitude mask apodization. J. Infrared Milli Terahz Waves 2018, 39, 546-552. [CrossRef]

52. Liu, C.; Chen, W.; Geints, Y.; Minin, O.V.; Minin, I.V. Simulation and experimental observations of axial position control of photonic nanojet by dielectric cube with metal screen. Opt. Lett. 2021, 46, 4292-4295. [CrossRef]

53. Liu, C.; Chen, W.; Minin, O.V.; Minin, I.V. Multispectral photonic jet shaping and steering by control of tangential electric field component on cuboid particle. Photonics 2021, 8, 317. [CrossRef]

54. Dorofeev, I.; Suslyaev, V.; Minin, O.V.; Minin, I.V. Role of the tangential electric field component to the terahertz jet and hook formation by dielectric cube and sphere. Opt. Eng. 2021, 60, 82004. [CrossRef]

55. Ling, J.; Li, D.; Liu, X.; Wang, X. Ultra-long focusing of microsphere lens via wavefront reconstruction in microsphere. Proc. SPIE 2018, 10964, 109642D.

56. Pacheco-Pena, V.; Beruete, M.; Minin, I.V.; Minin, O.V. Terajets produced by 3D dielectric cuboids. Appl. Phys. Lett. 2014, 105, 84102.

57. Zhao, L.; Ong, C.K. Direct observation of photonic jets and corresponding backscattering enhancement at microwave frequencies. J. Appl. Phys. 2009, 105, 123512. [CrossRef]

58. Pacheco-Pena, V.; Beruete, M.; Minin, I.V.; Minin, O.V. Multifrequency focusing and wide angular scanning of Terajets. Optic Lett. 2015, 40, 245. [CrossRef] 
59. Khodzitsky, M.; Vozianova, A.; Gill, V.; Chernyadiev, A.; Grebenchukov, A.; Minin, I.V.; Minin, O.V. Formation of terahertz beams produced by artificial dielectric periodical structures. Proc. SPIE 2016, 9918, 99182X.

60. Minin, I.V.; Minin, O.V.; Delgado-Notario, J.A.; Calvo-Gallego, J.; Velázquez-Pérez, J.E.; Ferrando-Bataller, M.; Meziani, Y.M. Improvement of a terahertz detector performance using the terajet effect in a mesoscale dielectric cube: Proof of concept. Phys. Status Solidi-Rapid Res. Lett. 2020, 14, 1900700. [CrossRef]

61. Minin, I.V.; Minin, O.V.; Salvador-Sanchez, J.; Delgado-Notario, J.A.; Calvo-Gallego, J.; Ferrando-Bataller, M.; Fobelets, K.; Velázquez-Pérez, J.E.; Meziani, Y.M. Responsivity enhancement of a strained silicon field effect transistor detector at $0.3 \mathrm{THz}$ using the terajet effect. Opt. Lett. 2021, 46, 3061-3064. [CrossRef] [PubMed]

62. Minin, O.V.; Minin, I.V.; Meziani, Y.M.; Hisatake, S. Improvement of a point-contact detector performance using the terajet effect initiated by photonics. Opt. Eng. 2020, 60, 82005. [CrossRef]

63. Dorofeev, I.; Dunaevsky, G.; Suslyaev, V.; Minin, I.V.; Minin, O.V. Small-sized body influence on the quality factor increasing of quasioptical resonator. Opt. Quantum Electron. 2017, 49, 355. [CrossRef]

64. Samura, Y.; Horio, K.; Antipov, V.B.; Shipilov, S.E.; Eremeev, A.I.; Minin, O.V.; Minin, I.V.; Hisatake, S. Characterization of mesoscopic dielectric cuboid antenna at millimeter-wave band. IEEE Antennas Wirel. Propag. Lett. 2019, 18, 1828-1832. [CrossRef]

65. Yamada, K.; Samura, Y.; Minin, O.V.; Kanno, A.; Sekine, N.; Nakajima, J.; Minin, I.V.; Hisatake, S. Short-range Wireless Transmission in the 300-GHz Band Using Low-profile Wavelength-scaled Dielectric Cuboid Antennas. Front. Comms. Net. 2021, 2, 702968. [CrossRef]

66. Wang, D.; Chen, X.; Fang, X.; Tang, J.; Lin, F.; Wang, X.; Liu, G.; Liao, L.; Ho, J.C.; Wei, Z. Photoresponse improvement of mixed-dimensional 1D-2D GaAs photodetectors by incorporating constructive interface states. Nanoscale 2021, 13, 1086-1092. [CrossRef] [PubMed]

67. Minin, I.V.; Minin, O.V.; Shuvalov, G.V. Dielectric wavelength-scaled metalenses based on an anomalous apodization effect for photoconductive optical-to-terahertz switches. In Proceedings of the 2021 XV International Conference on Actual Problems of Electronics Instrument Engineering (APEIE), Novosibirsk, Russia, 19-21 November 2021. accepted.

68. Gouy, L.G. Sur une propriete nouvelle des ondes lumineuses. C. R. Acad. Sci. Paris 1890, 110, 1251.

69. Kim, M.; Scharf, T.; Mühlig, S.; Rockstuhl, C.; Herzig, H. Gouy phase anomaly in photonic nanojets. Appl. Phys. Lett. 2011, 98, 191114. [CrossRef]

70. Bon, P.; Rolly, B.; Bonod, N.; Wenger, J.; Stout, B.; Monneret, S.; Rigneault, H. Imaging the Gouy phase shift in photonic jets with a wavefront sensor. Opt. Lett. 2012, 37, 3531-3533. [CrossRef]

71. Pham, H.; Hisatake, S.; Minin, I.V.; Minin, O.V.; Nagatsuma, T. Three-dimensional direct observation of Gouy phase shift in a terajet produced by a dielectric cuboid. Appl. Phys. Lett. 2016, 108, 191102. [CrossRef]

72. Pham, H.; Hisatake, S.; Minin, O.V.; Nagatsuma, T.; Minin, I.V. Asymmetric phase anomaly of terajet generated from dielectric cube under oblique illumination. Appl. Phys. Lett. 2017, 110, 201105. [CrossRef]

73. Liñares, J.; Prieto-Blanco, X.; Montero-Orille, C.; Moreno, N. Spatial mode multiplexing/demultiplexing by Gouy phase interferometry. Opt. Lett. 2017, 42, 93-96.

74. Christian, G.; Goubau, J. Experimental studies on a beam waveguide for millimeter waves. IRE Trans. Antennas Propag. 1961, 9 , 256. [CrossRef]

75. Sobel, F.; Wentworth, F.; Wiltse, J. Quasi-Optical Surface Waveguide and Other Components for the 100-to 300-Gc Region. IRE Trans. Microw. Theory Tech. 1961, 9, 512.

76. Chen, Z.; Taflove, A.; Backman, V. Highly efficient optical coupling and transport phenomena in chains of dielectric microspheres. Opt. Lett. 2006, 31, 389-391. [CrossRef]

77. Kapitonov, A.; Astratov, V. Observation of nanojet-induced modes with small propagation losses in chains of coupled spherical cavities. Opt. Lett. 2007, 32, 409-411. [CrossRef] [PubMed]

78. Yang, S.; Astratov, V. Photonic nanojet-induced modes in chains of size-disordered microspheres with an attenuation of only 0.08 dB per sphere. Appl. Phys. Lett. 2008, 92, 261111. [CrossRef]

79. Minin, I.V.; Minin, O.V.; Pacheco-Peña, V.; Beruete, M. All-dielectric periodic terajet waveguide using an array of coupled cuboids. Appl. Phys. Lett. 2015, 106, 254102. [CrossRef]

80. Mitsui, T.; Wakayama, Y.; Onodera, T.; Hayashi, T.; Ikeda, N.; Sugimoto, Y.; Takamasu, T.; Oikawa, H. Micro-demultiplexer of Coupled Resonator Optical Waveguide Fabricated by Microspheres. Adv. Mater. 2010, 22, 3022-3026. [CrossRef] [PubMed]

81. Mitsui, T.; Onodera, Y.; Wakayama, T.; Hayashi, N.; Ikeda, Y.; Sugimoto, T.; Takamasu, H.; Oikawa, H. Influence of micro-joints formed between spheres in coupled resonator optical waveguide. Opt. Express 2011, 19, 22258-22267. [CrossRef] [PubMed]

82. Liu, C.; Minin, O.V.; Minin, I.V. Periodical focusing mode achieved through a chain of mesoscale dielectric particles with a refractive index near unity. Opt. Commun. 2019, 434, 110-117. [CrossRef]

83. Mikaelyan, A. SELFOC dielectric waveguides. Sov. J. Quantum Electron. 1977, 7, 266-267. [CrossRef]

84. Liu, L.; Rahman, M.; Ning, Y.; Grattan, K. Accurate mode characterization of graded-index multimode fibers for the application of mode-noise analysis. Appl. Opt. 1995, 34, 1540-1543. [CrossRef]

85. Minin, I.V.; Minin, O.V.; Pacheco-Peña, V.; Beruete, M. Localized photonic jets from flat, three-dimensional dielectric cuboids in the reflection mode. Opt. Lett. 2015, 40, 2329-2332. [CrossRef] 
86. Minin, I.V.; Minin, O.V.; Kharitoshin, N. Localized High Field Enhancements from Hemispherical 3D Mesoscale Dielectric Particles in the Reflection Mode. In Proceedings of the 2015 16th International Conference of Young Specialists on Micro/Nanotechnologies and Electron Devices, Novosibirsk, Russia, 29 June-3 July 2015; pp. 331-333.

87. Minin, I.V.; Minin, O.V.; Nesedov, I. Photonic jets from Babinet's cuboid structures in the reflection mode. Opt. Lett. 2016, 41, 785-787. [CrossRef]

88. Yue, L.; Yan, B.; Monks, J.; Dhama, R.; Wang, Z.; Minin, O.V.; Minin, I.V. Photonic Jet by a Near-Unity-Refractive-Index Sphere on a Dielectric Substrate with High Index Contrast. Ann. Phys. 2018, 530, 1800032. [CrossRef]

89. Minin, I.V.; Geints, Y.; Zemlyanov, A.; Minin, O.V. Specular-reflection photonic nanojet: Physical basis and optical trapping application. Opt. Express 2020, 28, 22690. [CrossRef]

90. Wang, H.; Wu, X.; Shen, D. Trapping and manipulating nanoparticles in photonic nanojets. Opt. Lett. 2016, 41, 1652. [CrossRef]

91. Sergeeva, K.; Sergeev, A.; Minin, O.V.; Minin, I.V. A Closer Look at Photonic Nanojets in Reflection Mode: Control of Standing Wave Modulation. Photonics 2021, 8, 54. [CrossRef]

92. Minin, I.V.; Liu, C.; Yang, Y.; Staliunas, K.; Minin, O.V. Experimental observation of flat focusing mirror based on photonic jet effect. Sci. Rep. 2020, 10, 8459. [CrossRef]

93. Cheng, Y.; Kicas, S.; Trull, J.M.; Peckus, C.; Cojocaru, R.; Vilaseca, R. Drazdys \& K. Staliunas. Flat Focusing Mirror. Sci. Rep. 2014, 4, 6326. [PubMed]

94. Cheng, Y.; Staliunas, K. Near-field flat focusing mirrors. Appl. Phys. Rev. 2018, 5, 11101. [CrossRef]

95. Zhou, S.; Deng, Y.; Zhou, W.; Yu, M.; Urbach, H.; Wu, Y. Effects of whispering gallery mode in microsphere super-resolution imaging. Appl. Phys. B 2017, 123, 236. [CrossRef]

96. Hoang, T.; Duan, Y.; Chen, X.; Barbastathis, G. Focusing and imaging in microsphere-based microscopy. Opt. Express 2015, 23, 12337-12353. [CrossRef] [PubMed]

97. Geints, Y.; Panina, E.; Minin, I.V.; Minin, O.V. Study of focusing parameters of wavelength-scale binary phase Fresnel zone plate. J. Opt. 2021, 23, 65101. [CrossRef]

98. Minin, I.V.; Minin, O.V. Terahertz artificial dielectric cuboid lens on substrate for super-resolution images. Opt. Quant. Electron. 2017, 49, 326. [CrossRef]

99. Liu, C.; Yen, T.; Minin, O.V.; Minin, I.V. Engineering photonic nanojet by a graded-index micro-cuboid. Phys. E. Low-Dimens. Syst. Nanostructures 2018, 98, 105-110. [CrossRef]

100. Kwon, S.; Kim, K.; Park, J.; Cho, Y.; Lee, M. Microsphere-assisted ultra-small spot spectral reflectometry technique for semiconductor device metrology. Proc. SPIE 2021, 11611, 116110G.

101. Minin, I.V.; Minin, O.V.; Cao, Y.; Yan, B.; Wang, Z.; Luk'yanchuk, B. Photonic Lenses with whisphering Gallery Waves at Janus Particles. arXiv 2020, arXiv:2012.09489.

102. de Gennes, P.-G. Soft Matter (Nobel Lecture). Angew. Chem. Int. Ed. Engl. 1992, 31, 842-845. [CrossRef]

103. Mason, D.; Jouravlev, M.; Kim, S. Enhanced resolution beyond the Abbe diffraction limit with wavelength-scale solid immersion lenses. Opt. Lett. 2010, 35, 2007-2009. [CrossRef]

104. Kino, G.; Mansfield, S. Near field and solid immersion optical microscope. US Patent 5,004,307, 2 April 1991.

105. Yu, N.; Genevet, P.; Kats, M.; Aieta, F.; Tetienne, J.; Capasso, F.; Gaburro, Z. Light Propagation with Phase Discontinuities: Generalized Laws of Reflection and Refraction. Science 2011, 334, 333-337. [CrossRef]

106. Pham, H.; Hisatake, S.; Minin, O.V.; Nagatsuma, T.; Minin, I.V. Enhancement of Spatial Resolution of Terahertz Imaging Systems Based on Terajet Generation by Dielectric Cube. APL Photonics 2017, 2, 56106. [CrossRef]

107. Minin, I.V.; Minin, O.V.; Salvador-Sanchez, J.; Delgado-Notario, J.; Calvo-Gallego, J.; Baranov, P.; Ferrando-Bataller, M.; Fobelets, K.; Velazquez-Perez, J.; Meziani, Y. Imaging resolution enhancement using terajet effect at 0.3THz. Proc. SPIE 2021, 11868, $118680 \mathrm{~N}$.

108. Minin, I.V.; Minin, O.V. Terahertz Microscopy with Oblique Subwavelength Illumination in near Field. Preprints 2021, $2021,90024$. [CrossRef]

109. Abbe, E. On the estimation of the aperture of the microscope. J. Roy Microscop. Soc. Ser. II 1881, 1, 349-388. [CrossRef]

110. Sanchez, C.; Cristobal, G.; Bueno, G.; Blanco, S.; Borrego-Ramos, M.; Olenici, A.; Pedraza, A.; Ruiz-Santaquiteria, J. Oblique illumination in microscopy: A quantitative evaluation. Micron 2018, 105, 47-54. [CrossRef] [PubMed]

111. Minin, I.V.; Minin, O.V. THz microscopy with oblique subwavelength illumination: Proof of concept. Quantum Electron. 2022. accepted.

112. Cao, Y.; Liu, Z.; Minin, O.V.; Minin, I.V. Deep Subwavelength-Scale Light Focusing and Confinement in Nanohole-Structured Mesoscale Dielectric Spheres. Nanomaterials 2019, 9, 186. [CrossRef]

113. Minin, I.V.; Minin, O.V.; Cao, Y.; Liu, Z.; Geints, Y.E.; Karabchevsky, A. Optical vacuum cleaner by optomechanical manipulation of nanoparticles using nanostructured mesoscale dielectric cuboid. Sci. Rep. 2019, 9, 12748. [CrossRef]

114. Minin, I.V.; Minin, O.V. Ultrafast all-optical THz modulation based on wavelength scaled dielectric particle with graphene monolayer. Proc. SPIE 2019, 11065, 110651J.

115. Minin, O.V.; Minin, I.V.; Cao, Y. Optical magnet for nanoparticles manipulations based on optical vacuum cleaner concept. Proc. SPIE 2021, 11845, 118451G.

116. Zhou, Y.; Hong, M. Formation of a three-dimensional bottle beam via an engineered microsphere. Photonics Res. 2021, 9, 1598. [CrossRef] 
117. Dholakia, K.; Bruce, G. Optical hooks. Nat. Photonics 2019, 13, 229. [CrossRef]

118. Minin, I.V.; Minin, O.V. Recent Trends in Optical Manipulation Inspired by Mesoscale Photonics and Diffraction Optics. J. Biomed. Photonics Eng. 2020, 6, 20301. [CrossRef]

119. Minin, I.V.; Minin, O.V. Device for forming the optical trap in the form of the photonic hook. Patent of Russia 161207,27 October 2015.

120. Yue, L.; Minin, O.V.; Wang, Z.; Monks, J.; Snalin, A.; Minin, I.V. Photonic hook: A new curved light beam. Opt. Lett. $2018,43,771$. [CrossRef]

121. Spector, M.; Ang, A.; Minin, O.V.; Minin, I.V.; Karabchevsky, A. Temperature mediated 'Photonic hook' nanoparticle manipulator with pulsed illumination. Nanoscale Adv. 2020, 2, 2595-2601. [CrossRef]

122. Minin, I.V.; Minin, O.V. The Photonic Hook: From Optics to Acoustics and Plasmonics; Springer: Cham, Germany, 2021.

123. Minin, I.V.; Minin, O.V.; Katyba, G.; Chernomyrdin, N.; Zaytsev, K.; Yue, L.; Wang, Z.; Christodoulides, D.N. Experimental observation of a photonic hook. APL 2019, 114, 31105. [CrossRef]

124. Spector, M.; Ang, A.; Minin, O.V.; Minin, I.V.; Karabchevsky, A. Photonic hook formation in near-infrared with MXene Ti3C2 nanoparticles. Nanoscale Adv. 2020, 2, 5312-5318. [CrossRef]

125. Minin, I.V.; Minin, O.V. Dielectric particle-based strategy to design a new self-bending subwavelength structured light beams. IOP Conf. Series Mater. Sci. Eng. 2021, 1019, 12093. [CrossRef]

126. Geints, Y.; Minin, I.V.; Minin, O.V. Tailoring 'photonic hook' from Janus dielectric microbar. J. Opt. 2020, 22, 65606. [CrossRef]

127. Gu, G.; Zhang, P.; Chen, S.; Zhang, Y.; Yang, H. Inflection point: A perspective on photonic nanojets. Photonics Res. 2021, 9 , 1157. [CrossRef]

128. Minin, I.V.; Minin, O.V.; Liu, C.; Wei, H.; Geints, Y.; Karabchevsky, A. Experimental demonstration of tunable photonic hook by partially illuminated dielectric microcylinder. Opt. Lett. 2020, 45, 4899. [CrossRef]

129. Liu, C.; Chung, H.; Minin, O.V.; Minin, I.V. Shaping photonic hook via well-controlled illumination of finite-size graded-index micro-ellipsoid. J. Opt. 2020, 22, 85002. [CrossRef]

130. Pacheco-Peña, V.; Riley, J.; Liu, C.; Minin, O.V.; Minin, I.V. Diffraction limited photonic hook via scattering and diffraction of dual-dielectric structures. Sci. Rep. 2021, 11, 20278. [CrossRef] [PubMed]

131. Ang, A.; Karabchevsky, A.; Minin, I.V.; Minin, O.V.; Sukhov, S.; Snalin, A. 'Photonic Hook' based optomechanical nanoparticle manipulator. Sci. Rep. 2018, 8, 2029. [CrossRef]

132. Ang, A.; Minin, I.V.; Minin, O.V.; Sukhov, S.; Snalin, A.; Karabchevsky, A. Low-contrast photonic hook manipulator for cellular differentiation. In Proceedings of the 9th International Conference on Metamaterials, Photonic Crystals and Plasmonics, META 2018, Marseille, France, 24 June-1 July 2018.

133. Cipolla, M.J. Barriers of the CNS. In The Cerebral Circulation. San Rafael (CA): Morgan \& Claypool Life Sciences; NCBI: Bethesda, ML, USA, 2009; Chapter 6. Available online: www.ncbi.nlm.nih.gov/books/NBK53084/ (accessed on 15 December 2021).

134. Minin, I.V.; Minin, O.V.; Liu, Y.; Tuchin, V.; Liu, C. Concept of Photonic Hook Scalpel Generated by Shaped Fiber Tip with Asymmetric Radiation. J. Biophotonics 2021, 14, e202000342. [CrossRef] [PubMed]

135. Geints, Y.; Minin, O.V.; Yue, L.; Minin, I.V. Wavelength-scale photonic space switch proof-of-concept based on photonic hook effect. Ann. Phys. 2021, 533, 2100192. [CrossRef]

136. Minin, I.V.; Shuvalov, G.V.; Minin, O.V. All-dielectric asymmetrical metasurfaces based on mesoscale dielectric particles with different optical transmissions in opposite directions through full internal reflection. In Frontier Research and Innovation in Optoelectronics Technology and Industry; CRC Press: Kunming, China, 2018; pp. 437-440.

137. Liu, C.-Y.; Minin, O.V.; Minin, I.V. First experimental observation of array of photonic jets from saw-tooth phase diffraction grating. EPL Europhys. Lett. 2018, 123, 54003. [CrossRef]

138. Ge, S.; Liu, W.; Zhang, J.; Huang, Y.; Xi, Y.; Yang, P.; Sun, X.; Li, S.; Lin, D.; Zhou, D.; et al. Novel Bilayer Micropyramid Structure Photonic Nanojet for Enhancing a Focused Optical Field. Nanomaterials 2021, 11, 2034. [CrossRef]

139. Balezin, M.; Baryshnikova, K.V.; Kapitanova, P.; Evlyukhin, A. Electromagnetic properties of the Great Pyramid: First multipole resonances and energy concentration. J. Appl. Phys. 2018, 124, 34903. [CrossRef]

140. Minin, I.V.; Minin, O.V.; Yue, L. Electromagnetic properties of Pyramids from positions of photonics. Russ. Phys. J. 2020, 62, 1763-1769. [CrossRef]

141. Schuller, J.; Barnard, E.; Cai, W.; Jun, Y.; White, J.; Brongersma, M. Plasmonics for extreme light concentration and manipulation. Nat. Mater. 2010, 9, 193. [CrossRef] [PubMed]

142. Zhao, C.; Zhang, J.; Liu, Y. Light manipulation with encoded plasmonic nanostructures. EPJ Appl. Metamaterials 2014, 1, 6. [CrossRef]

143. Ju, D.; Pei, H.; Jiang, Y.; Sun, Y. Controllable and enhanced nanojet effects excited by surface plasmon polariton. Appl. Phys. Lett. 2013, 102, 171109. [CrossRef]

144. Pacheco-Pena, V.; Minin, I.V.; Minin, O.V.; Beruete, M. Comprehensive analysis of photonic nanojets in 3D dielectric cuboids excited by surface plasmons. Ann. Phys. 2016, 528, 684. [CrossRef]

145. Pacheco-Pena, V.; Minin, I.V.; Minin, O.V.; Beruete, M. Increasing Surface Plasmons Propagation via Photonic Nanojets with Periodically Spaced 3D Dielectric Cuboids. Photonics 2016, 3, 10. [CrossRef]

146. Minin, I.V.; Minin, O.V.; Glinskiy, I.; Malureanu, R.; Lavrinenko, A.; Yakubovsky, D.; Volcov, V.; Ponomarev, D. Plasmonic nanojet: An experimental demonstration. Opt. Lett. 2020, 45, 3244-3247. [CrossRef] 
147. Caldwell, J.; Lindsay, L.; Giannini, V.; Vurgaftman, I.; Reinecke, T.; Maier, S.; Glembocki, O. Low-loss, infrared and terahertz nanophotonics using surface phonon polaritons. Nanophotonics 2015, 4, 44-68. [CrossRef]

148. Minin, I.V.; Minin, O.V.; Ponomarev, D.; Glinskiy, I. Photonic hook plasmons: A new curved surface wave. Ann. Phys. 2018, 530, 1800359. [CrossRef]

149. Minin, I.V.; Minin, O.V.; Glinskiy, I.; Malureanu, R.; Lavrinenko, A.; Yakubovsky, D.; Volcov, V.; Ponomarev, D. Experimental verification of a plasmonic hook in a dielectric Janus particle. APL 2021, 118, 131107. [CrossRef]

150. Zhang, Y.; Min, C.; Dou, X.; Wang, X.; Urbach, H.; Somekh, M.; Yuan, X. Plasmonic tweezers: For nanoscale optical trapping and beyond. Light Sci. Appl. 2021, 10, 59. [CrossRef]

151. Minin, O.V.; Minin, I.V. Acoustojet: Acoustic analogue of photonic jet phenomenon based on penetrable 3D particle. Opt. Quantum Electron. 2017, 49, 54. [CrossRef]

152. Lopes, J.; Andrade, M.; Leao-Neto, J.; Adamowski, J.; Minin, I.V.; Silva, G. Focusing acoustic beams with a ball shaped lens beyond the diffraction limit. Phys. Rev. Appl. 2017, 8, 24013. [CrossRef]

153. Minin, O.V.; Minin, I.V. Mesoscale acoustical cylindrical superlens. MATEC Web Conf. 2018, 155, 1029. [CrossRef]

154. Tarrazo-Serrano, D.; Rubio, C.; Minin, O.V.; Uris, A.; Minin, I.V. Ultrasonic focusing with mesoscale polymer cuboid. Ultrasonics 2020, 106, 106143. [CrossRef] [PubMed]

155. Zhao, L.; Horiuchi, T.; Yu, M. Broadband ultra-long acoustic jet based on double-foci Luneburg lens. JASA Express Lett. 2021, 1, 114001. [CrossRef]

156. Rubio, C.; Tarrazo-Serrano, D.; Minin, O.V.; Uris, A.; Minin, I.V. Enhancement of pupil masked wavelength-scale gas-filled flat acoustic lens based on anomaly apodization effect. Phys. Lett. A 2019, 383, 396-399. [CrossRef]

157. Lu, C.; Yu, R.; Wang, K.; Wang, J.; and Wu, D. Tunable acoustic jet generated by a masked cylindrical lens. Appl. Phys. Express 2020, 13, 97003. [CrossRef]

158. Minin, I.V.; Minin, O.V.; Tseplyaev, I. The relationship between resonance scattering and the formation of an acoustojet under the interaction of ultrasound with a dielectric sphere immersed in water. J. Phys. Conf. Ser. 2017, 881, 12025. [CrossRef]

159. Rubio, C.; Minin, I.V.; Uris, A.; Minin, O.V. Super-resonances in a dielectric mesoscale sphere immersed in water: Effects in extreme field localization of acoustic wave. Proc. Mtgs. Acoust. 2019, 38, 70001.

160. Rubio, C.; Tarrazo-Serrano, D.; Minin, O.V.; Uris, A.; Minin, I.V. Acoustical hooks: A new subwavelength self-bending beam. Results Phys. 2020, 16, 102921. [CrossRef]

161. Ren, X.; Zhou, Q.; Xu, Z.; Liu, X. Acoustic hook beam lens for particle trapping. Appl. Phys. Express 2020, 13, 64003. [CrossRef]

162. Castiñeira-Ibáñez, S.; Tarrazó-Serrano, D.; Uris, A.; Rubio, C.; Minin, O.V.; Minin, I.V. Cylindrical 3D printed configurable ultrasonic lens for subwavelength focusing enhancement. Sci. Rep. 2020, 10, 20279. [CrossRef]

163. Castiñeira-Ibáñez, S.; Tarrazó-Serrano, D.; Uris, A.; Rubio, C. Tunable acoustic hooks from Janus cylinder. Results Phys. 2021, 24, 104134. [CrossRef]

164. Sarvazyan, A.; Urban, M.; Greenleaf, J. Acoustic waves in medical imaging and diagnostics. Ultrasound Med. Biol. 2013, 39, 1133-1146. [CrossRef] [PubMed]

165. Gorgin, R.; Luo, Y.; Wu, Z. Environmental and operational conditions effects on Lamb wave based structural health monitoring systems: A review. Ultrasonics 2020, 105, 106114. [CrossRef]

166. Wang, J.; Yurchenko, D.; Hu, G.; Zhao, L.; Tang, L.; Yang, Y. Perspectives in flow-induced vibration energy harvesting. Appl. Phys. Lett. 2021, 119, 100502. [CrossRef]

167. Taylor, M.A.; Waleed, M.; Stilgoe, A.B.; Rubinsztein-Dunlop, H.; Bowen, W.P. Enhanced optical trapping via structured scattering. Nat. Photon. 2015, 9, 669-673. [CrossRef]

168. Dholakia, K.; Čižmár, T. Shaping the future of manipulation. Nat. Photon. 2011, 5, 335-342. [CrossRef]

169. Long, L.; Chen, J.; Yu, H.; Li, Z.Y. Strong optical force of a molecule enabled by the plasmonic nanogap hot spot in a tip-enhanced Raman spectroscopy system. Photon. Res. 2020, 8, 1573-1579. [CrossRef]

170. Shi, L.; Meseguer, F. Magnetic interaction in all silicon waveguide spherical coupler device. Opt. Express. 2012, 20, 22616-22626. [CrossRef] [PubMed]

171. Rastinehad, A.; Anastos, H.; Wajswol, E.; Winoker, J.S.; Sfakianos, J.P.; Doppalapudi, S.K.; Carrick, M.R.; Knauer, C.J.; Taouli, B.; Lewis, S.C.; et al. Gold nanoshell-localized photothermal ablation of prostate tumors in a clinical pilot device study. PNAS 2019, 116, 18590-18596. [CrossRef]

172. Chen, L.; Zhou, Y.; Zhou, R.; Hong, M. Microsphere-Toward Future of Optical Microscopes. iScience 2020, 23, 101211. [CrossRef]

173. Savo, R.; Morandi, A.; Muller, J.S.; Kaufmann, F.; Timpu, F.; Escale, M.R.; Zanini, M.; Isa, L.; Grange, R. Broadband Mie driven random quasi-phasematching. Nat. Photonics 2020, 14, 740-747. [CrossRef]

174. Huang, K.; Ye, H.; Teng, J.; Yeo, S.P.; Luk'yanchuk, B.; Qiu, C.W. Optimization-free superoscillatory lens using phase and amplitude masks. Laser Photonics Rev. 2014, 8, 152-157. [CrossRef]

175. Minin, I.V.; Minin, O.V. Control of focusing properties of diffraction elements. Sov. J. Quantum. Electron. 1990, 20, 198-199. [CrossRef]

176. Mahariq, I.; Giden, I.; Minin, I.V.; Minin, O.V.; Kurt, H. Strong electromagnetic field localization near the surface of hemicylindrical particles. Opt. Quant. Electron. 2017, 49, 423. [CrossRef]

177. Minin, I.V.; Minin, O.V.; Eremeev, A.; Tseplyaev, I. Spherical and cylindrical particle resonator as a cloak system. IOP Conf. Series Mater. Sci. Eng. 2018, 363, 12026. [CrossRef] 
178. Sugathan, B.; Nilaya, J.P.; Pillai, V.; Biswas, D.J. Particle assisted structuring on metallic substrate: Anomaly when particle size exceeds irradiation wavelength. AIP Adv. 2020, 10, 35222. [CrossRef]

179. Kravtsov, Y.A.; Orlov, Y.I. Limits of applicability of the method of geometric optics and related problems. Sov. Phys. Usp. 1980, 23, 750. [CrossRef] 